\title{
The evolution of the temperature field during cavity collapse in liquid nitromethane. Part II: reactive case
}

\author{
L. Michael ${ }^{1}$ (I) N. Nikiforakis ${ }^{1}$
}

Received: 5 September 2017 / Revised: 27 November 2017 / Accepted: 4 January 2018 / Published online: 10 February 2018

(C) The Author(s) 2018. This article is an open access publication

\begin{abstract}
This work is concerned with the effect of cavity collapse in non-ideal explosives as a means of controlling their sensitivity. The main objective is to understand the origin of localised temperature peaks (hot spots) which play a leading order role at the early stages of ignition. To this end, we perform two- and three-dimensional numerical simulations of shock-induced single gas-cavity collapse in liquid nitromethane. Ignition is the result of a complex interplay between fluid dynamics and exothermic chemical reaction. In the first part of this work, we focused on the hydrodynamic effects in the collapse process by switching off the reaction terms in the mathematical formulation. In this part, we reinstate the reactive terms and study the collapse of the cavity in the presence of chemical reactions. By using a multi-phase formulation which overcomes current challenges of cavity collapse modelling in reactive media, we account for the large density difference across the material interface without generating spurious temperature peaks, thus allowing the use of a temperature-based reaction rate law. The mathematical and physical models are validated against experimental and analytic data. In Part I, we demonstrated that, compared to experiments, the generated hot spots have a more complex topological structure and that additional hot spots arise in
\end{abstract}

Communicated by D. Zeidan and H. D. Ng.

L. Michael

1m355@cam.ac.uk

1 Laboratory for Scientific Computing, Cavendish Laboratory, Department of Physics, University of Cambridge, Cambridge, UK regions away from the cavity centreline. Here, we extend this by identifying which of the previously determined hightemperature regions in fact lead to ignition and comment on the reactive strength and reaction growth rate in the distinct hot spots. We demonstrate and quantify the sensitisation of nitromethane by the collapse of the isolated cavity by comparing the ignition times of nitromethane due to cavity collapse and the ignition time of the neat material. The ignition in both the centreline hot spots and the hot spots generated by Mach stems occurs in less than half the ignition time of the neat material. We compare two- and three-dimensional simulations to examine the change in topology, temperatures, and reactive strength of the hot spots by the third dimension. It is apparent that belated ignition times can be avoided by the use of three-dimensional simulations. The effect of the chemical reactions on the topology and strength of the hot spots in the timescales considered is also studied, in a comparison between inert and reactive simulations where maximum temperature fields and their growth rates are examined.

Keywords Condensed-phase explosives - Cavity collapse . Temperature $\cdot$ Nitromethane $\cdot$ Hot spots $\cdot$ Ignition

\section{Introduction}

This work is motivated by the necessity for optimising the performance of non-ideal, inhomogeneous explosives such as those used in mining. In order to increase their sensitivity and to control their performance, cavities are introduced in the bulk of the explosive, often by means of glass microballoons. When a precursor shock wave passes through the explosive, these cavities collapse, generating regions of locally high pressure and temperature, which are commonly referred to as hot spots. These lead to multiple local ignition 
sites, which cumulatively result in a shorter time to ignition than that of the neat material. Parameters such as the number, size, shape, and distribution of the cavities affect the degree of sensitisation of the explosive. Understanding the correlation between these parameters and the reduced ignition time will allow better control of the behaviour of the explosive.

To this end, the collapse of cavities has been extensively studied in the past by means of experiment and numerical simulation. An extensive literature review on previous studies in inert materials is given in Part I of this work [1]. Hence, here we indicatively only mention some of the studies for cavities collapsing in reactive liquid, gelatinous, and solid explosives. To determine the effect of the collapse on the hot spot generation and explosive ignition, cavity collapse experiments in reactive materials were performed by Bourne and Field $[2,3]$. The principal ignition mechanism was determined to be the hydrodynamic heating due to jet impact. Conduction from the highly compressed, heated gas inside the cavity does not occur in the short timescales governing the particular shock wave-cavity configurations studied.

Limitation of computational power restricted early numerical work on cavity collapse in reactive materials. Among the first studies on cavity collapse in a reactive medium was the work by Bourne and Milne [4,5] who observed numerically the same loci of hot spots as in experiments. More recently, the authors presented limited results on simulating cavity collapse in reacting nitromethane [6]. Kapila et al. [7] presented the collapse process and the detonation generation in an exemplary explosive and studied the effect of cavity shape in the detonation generation using a pressuredependent reaction rate. In an elastoplastic framework, Tran and Udaykumar [8] studied the response of reactive HMX with micron-sized cavities, while Rai et al. $[9,10]$ considered the resolution required for reactive cavity collapse simulations and the sensitivity behaviour of elongated cavities in HMX.

Despite technological advances, performing complete numerical simulations of ignition due to shock-induced cavity collapse still poses many challenges. These include the use of complex equations of state to describe the explosive materials, maintaining oscillation-free pressure, velocity, and temperature fields across the cavities material boundaries upon their interaction with shock waves, sustaining (at least) 1000:1 density difference across these boundaries, retrieving physically accurate temperature fields in the explosive matrix, and numerically modelling the ignition of the material as a temperature-driven phenomenon. Moreover, the computational power needed for accurately resolving the complete phenomenon in three dimensions is still large. As the explosive initiation is a temperature-driven phenomenon, the challenges regarding the temperature field are of critical importance. These challenges are described in detail in Part I of this work.
A complete physical simulation of the initiation of a condensed-phase explosive due to cavity collapse has several requirements: a three-dimensional framework, realistic material models (equations of state), oscillation-free material interfaces, the ability to recover accurate and oscillation-free temperature fields. A temperature-dependent reaction rate law is also desirable, to mathematically describe ignition to be driven by the heating of the material. Moreover, each component used should be validated alone and in combination with all the components composing the numerical framework.

Simulations presented in the literature satisfy some but not all of these requirements. In this work, we exercise the mathematical model (MiNi16) proposed by the authors in previous work [11] to overcome the difficulties in numerically simulating the cavity collapse and move towards a complete simulation of explosive initiation due to cavity collapse. In Part I of this work [1], we simulated the three-dimensional collapse of isolated air cavities in nitromethane using a validated equation of state (Cochran-Chan). We looked in depth how the hydrodynamical effects in the absence of reaction (e.g., generation and propagation of waves) lead to local temperature elevation. Such regions were identified as candidates for critical hot spots in a reactive simulation, and the necessity for three-dimensional (as opposed to two-dimensional) simulation was justified. In this second part of the work, we take advantage of oscillation-free and reliable temperature fields that can be recovered by using the MiNi16 model and extend the work of Part I to reactive scenarios. We perform three-dimensional simulations of the collapse of isolated air cavities in reacting, liquid nitromethane, using equations of state in Mie-Grüneisen form and a temperature-dependent reaction rate law. We study in detail the ignition process, and we link the evolution of the reaction progress variable to the temperature elevations and the wave pattern generated during the collapse process. We identify the reacting hot spots and study their relative reactive strength and reaction growth rates. The effect of the cavity collapse on shortening the time to ignition is illustrated explicitly by comparing the ignition due to cavity collapse against the ignition of the neat material. We also demonstrate the necessity for three-dimensional simulations (compared to 2D) by looking at the percentage of burnt material over time in the two scenarios but also looking at the evolution of waves and temperature fields. Moreover, we compare inert and reactive simulations to examine the added effect of the reactions on the temperature fields and the topology of the hot spots at the timescales considered.

The rest of the paper is outlined as follows: the next section presents the underlying mathematical formulation in terms of the governing partial differential equations, the equations of state that close the system, and the form and calibration of the reaction rate law for nitromethane combustion. A section on validation follows, where we compare numerical results 
against theoretical and experimental temperatures in shocked nitromethane and the CJ and von Neumann values for steadystate detonation. The ignition regime is validated in a similar way, by comparing numerical and experimental times-toignition for various input pressures with the use of an ignition Pop plot. In the results section, we consider the collapse of a gas cavity in liquid nitromethane, follow the events leading to the generation of local temperatures which are more than three times the post-shock temperature of the neat material and compare and analyse the difference between the 2D and $3 \mathrm{D}$ simulations as well as inert and reactive simulations in the context mentioned earlier.

\section{Mathematical and physical models}

A formulation that rectifies the issues commonly presented in shock-bubble simulations was proposed by Michael and Nikiforakis [11]. This formulation considers the cavity as an inert phase (phase 1) and the surrounding material as a reacting phase (phase 2) composed of two materials; the reactant nitromethane as material $\alpha$ and the gaseous products of reaction as material $\beta$. Mixing rules are employed to determine the properties of phase 2 from the properties of the two materials. Mixing rules are also in effect between phases 1 and 2 across material interfaces where the diffusion zones lie. In this work, we neglect the effect of reaction products and thus use a reduced form of the formulation.

Consider the gas inside the cavity to be phase 1 and the liquid nitromethane around the cavity to be phase 2 . Then, the governing equations for this system take the form:

$$
\begin{aligned}
& \frac{\partial z_{1} \rho_{1}}{\partial t}+\nabla \cdot\left(z_{1} \rho_{1} \mathbf{u}\right)=0, \\
& \frac{\partial z_{2} \rho_{2}}{\partial t}+\nabla \cdot\left(z_{2} \rho_{2} \mathbf{u}\right)=0, \\
& \frac{\partial}{\partial t}\left(\rho \mathbf{u}_{\mathbf{k}}\right)+\nabla \cdot\left(\rho \mathbf{u}_{\mathbf{k}} \mathbf{u}\right)+\frac{\partial p}{\partial \mathbf{x}_{\mathbf{k}}}=0, \\
& \frac{\partial}{\partial t}(\rho E)+\nabla \cdot[(\rho E+p) \mathbf{u}]=0, \\
& \frac{\partial z_{1}}{\partial t}+\mathbf{u} \cdot \nabla z_{1}=0, \\
& \frac{\partial z_{2} \rho_{2} \lambda}{\partial t}+\nabla \cdot\left(z_{2} \rho_{2} \mathbf{u} \lambda\right)=z_{2} \rho_{2} K,
\end{aligned}
$$

where for $i=1,2, \rho_{i}$ are the densities for the air and nitromethane, $z_{i}$ are their corresponding volume fractions $\left(z_{1}+z_{2}=1\right), \rho$ is the total density given by $\rho=z_{1} \rho_{1}+z_{2} \rho_{2}$, and $\mathbf{u}$ is the velocity vector and $p$ is the total pressure. The total specific energy is given by $E=\frac{1}{2} u^{2}+e$, where $e$ is the total specific internal energy. Also, $\lambda$ is a reaction progress variable and $K$ represents the reaction source terms, to be defined later. The mixture rule for the total internal energy is given by $\rho e=\rho_{1} z_{1} e_{1}+\rho_{2} z_{2} e_{2}$, where $e_{i}$ for $i=1,2$ are the specific internal energies for the air and nitromethane, given by their corresponding equations of state. A mixture rule for $\xi=\frac{1}{\gamma-1}$, where $\gamma$ is the total adiabatic index, is also required and in this case is given by $\xi=z_{1} \xi_{1}+z_{2} \xi_{2}$. The sound speed for the total mixture is given by

$\xi c^{2}=Y_{1} \xi_{1} c_{1}^{2}+Y_{2} \xi_{2} c_{2}^{2}$

where $Y_{i}$ is the mass fraction of phase $i$, given by $Y_{i}=\frac{\rho_{i} z_{i}}{\rho}$, for $i=1,2$.

This formulation can be considered an augmented twophase model for condensed-phase explosives in the same way that the Euler equations have been augmented to study gaseous combustion problems [12].

The nitromethane is modelled by the Cochran-Chan equation of state as presented in Part I of this work. In reactive simulations, a term $Q$ is included in the reference energy function of the reactant, representing the heat of detonation released upon reaction, such that $e_{\text {ref }}=e_{\text {ref }}+Q$. Alternatively, this term can be incorporated as a source term to the energy equation.

\subsection{Equations of state}

To close the system, the Cochran-Chan equation of state [13] is employed to describe the liquid nitromethane. This is an equation of state of Mie-Grüneisen form and is given by

$p(\rho, e)=p_{\mathrm{ref}}(\rho)+\rho \Gamma(\rho)\left[e-e_{\mathrm{ref}}(\rho)\right]$,

with reference pressure given by

$p_{\text {ref }}(\rho)=\mathcal{A}\left(\frac{\rho_{0}}{\rho}\right)^{-\mathcal{E}_{1}}-\mathcal{B}\left(\frac{\rho_{0}}{\rho}\right)^{-\mathcal{E}_{2}}$,

reference energy given by

$$
\begin{aligned}
e_{\mathrm{ref}}(\rho)= & \frac{-\mathcal{A}}{\rho_{0}\left(1-\mathcal{E}_{1}\right)}\left[\left(\frac{\rho_{0}}{\rho}\right)^{1-\mathcal{E}_{1}}-1\right] \\
& +\frac{\mathcal{B}}{\rho_{0}\left(1-\mathcal{E}_{2}\right)}\left[\left(\frac{\rho_{0}}{\rho}\right)^{1-\mathcal{E}_{2}}-1\right],
\end{aligned}
$$

and Grüneisen coefficient $\Gamma(\rho)=\Gamma_{0}$. The gas inside the cavity is modelled by the ideal gas equation of state, which is of Mie-Grüneisen form as well, with $p_{\text {ref }}=0$ and $e_{\text {ref }}=0$. The parameters for the equations of state of the two materials are given in Table 1. 
Table 1 Equation of state parameters for nitromethane and air

\begin{tabular}{llllllll}
\hline Equation of state parameters & $\Gamma_{0}(-)$ & $\mathcal{A}(\mathrm{GPa})$ & $\mathcal{B}(\mathrm{GPa})$ & $\mathcal{E}_{1}(-)$ & $\mathcal{E}_{2}(-)$ & $\rho_{0}\left(\mathrm{~kg} \mathrm{~m}^{-3}\right)$ & $c_{v}\left(\mathrm{~J} \mathrm{~kg}^{-1} \mathrm{~K}^{-1}\right)$ \\
\hline Nitromethane [13] & 1.19 & 0.819 & 1.51 & 4.53 & 1.42 & 1134 & 1714 \\
Air & 0.4 & - & - & - & - & - & 718 \\
\hline
\end{tabular}

\subsection{Recovery of temperature}

The multi-phase nature of the model allows for separate temperature fields to be computed for each material as

$T_{i}=\frac{p-p_{\text {ref }_{i}}(\rho)}{\rho_{i} \Gamma_{i} c_{v_{i}}}$, for $i=1,2$.

As a result, the nitromethane temperature $\left(T_{\mathrm{NM}}=T_{2}\right)$ is computed explicitly from the equation of state and can be used directly in the reaction rate law. For more information on the temperature recovery, the reader is referred to Part I.

\subsection{Reaction rates for nitromethane}

In order to model the reactions in nitromethane, a single-step, temperature-dependent Arrhenius reaction rate law is used, of the form

$K=\frac{\mathrm{d} \lambda}{\mathrm{d} t}=-\lambda C \mathrm{e}^{-T_{\mathrm{A}} / T_{\mathrm{NM}}}$,

where $C$ is a constant pre-exponential factor and $T_{\mathrm{A}}$ is the activation temperature of the material.

Many sets of the reaction rate parameters are available in the literature for liquid nitromethane. For example, $\left(C, T_{\mathrm{A}}\right)=\left(2.6 \times 10^{9} \mathrm{~s}^{-1}, 11,500 \mathrm{~K}\right)$ is suggested by Hardesty [14], $\left(6.9 \times 10^{10} \mathrm{~s}^{-1}, 14,400 \mathrm{~K}\right)$ is used by Tarver and Urtiew [15] and $\left(1.27 \times 10^{12} \mathrm{~s}^{-1}, 20,110 \mathrm{~K}\right)$ is used by Ripley et al. [16]. In this work, we adopt the pre-exponential factor proposed by Hardesty [14] and adjust the activation temperature to $T_{\mathrm{A}}=11,350 \mathrm{~K}$ to match the experimentally calculated overtake time and the shape of the velocity versus distance graph of the shock-induced ignition experiment in Sheffield et al. [17] (neat nitromethane, shocked at 9.1 GPa). It should be noted that even though the single-step Arrhenius rate equation is a good starting point for investigating general trends associated with hot spot ignition and burn [18], care should be taken with regard to its pressure validity regime, as suggested (for pressures of 0.1-5GPa) by Shaw et al. [19]. In this work, the parameters have been calibrated and validated for the range of pressures arising in the problem at hand, but adjustment might be needed if other regimes are considered. Moreover, it should be noted that multi-step models exist, such as, for example, the two-stage model by Kipp and Nunziato [20] developed for simultaneous modelling ignition and detonation regimes, as well as second-order single-step models, as used, for example, by Menikoff and Shaw [21], and they might have a quantitative effect on the temperature evolution.

In order to calculate the value for the heat of detonation, $Q$, we follow the approach described by Arienti et al. [22]. This involves varying the parameter $Q$ to match as closely as possible the experimental value of the pressure at the $\mathbf{C J}$ point of $12.5 \mathrm{GPa}$ [23] on the $p-v$ plane. In general, varying the value of $Q$ shifts the reactive Hugoniots upwards. When the Rayleigh line becomes tangent to the reactive (pseudoproduct) Hugoniot, the appropriate value for $Q$ is determined. Here, $Q=4.48 \times 10^{6} \mathrm{~J} \mathrm{~kg}^{-1}$.

We note that the set of calibrated parameters given above is valid for steady-state detonation propagation of neat nitromethane, and no further adjustment is necessary for ignition case studies, as the results in the validation section indicate.

\section{Validation}

System (1) is integrated numerically using a high-resolution shock-capturing numerical scheme, namely the MUSCLHancock finite volume method with an underlying HLLC Riemann solver. The non-conservative $z_{1}$ equation is handled following the work by Saurel et al. [13]. More information on the mathematical model and the numerical method used is given in [11]. Hierarchical, structured, adaptive mesh refinement (AMR) is used to dynamically increase the resolution locally [24]. The source terms that describe chemical reactions are integrated with a high-order Runge-Kutta scheme.

The aim of this section is to validate the resulting code and also to assess whether the combination of parameters described in the previous section can match the experimentally determined von Neumann spike, the CJ values, and the ignition Pop plot, without any further user adjustment. The inert formulation and physical model were validated in Part I of this work, showing non-oscillatory hydrodynamic fields and recovery of realistic temperature fields in shocked nitromethane.

\subsection{Steady ZND detonation}

In Part I, we validated the post-shock temperature field. Here, we assess whether the code predicts the correct CJ and von 


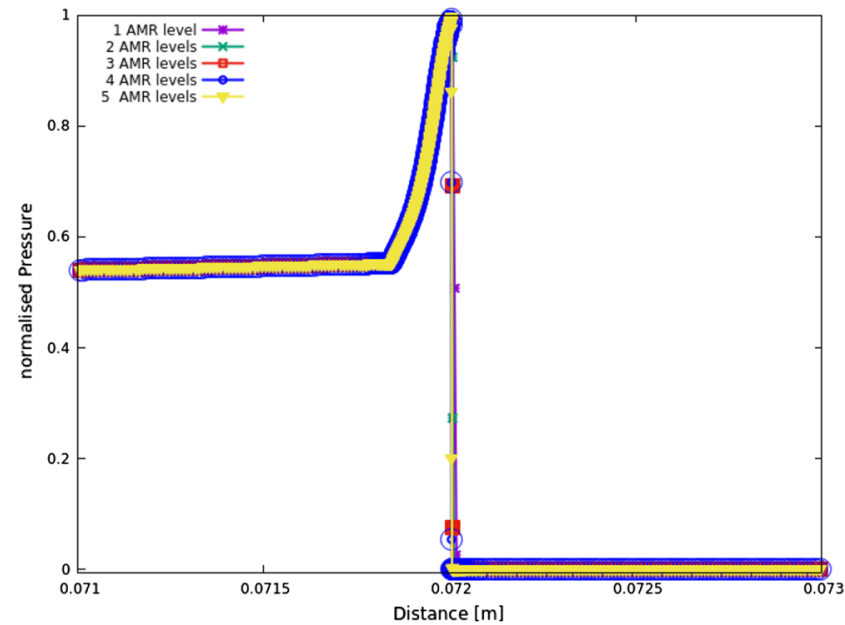

(a)

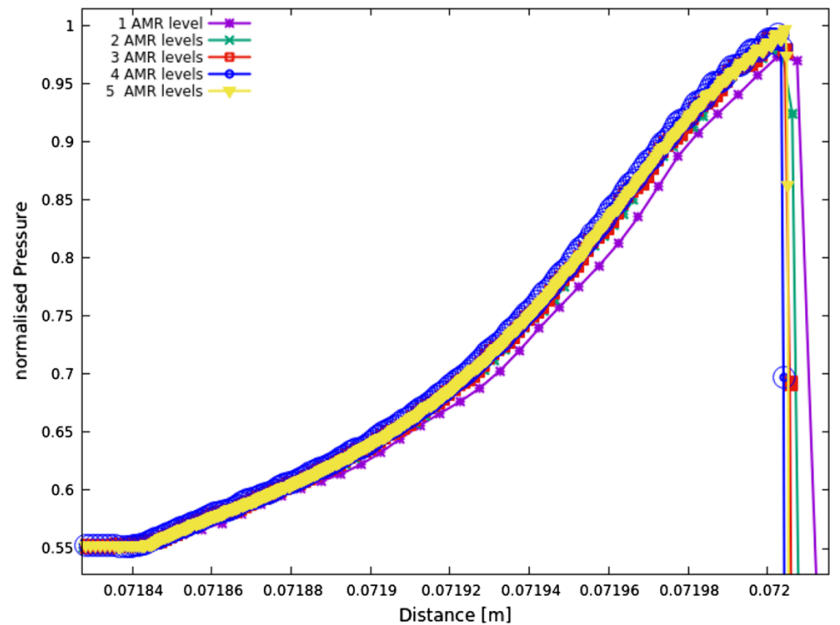

(b)

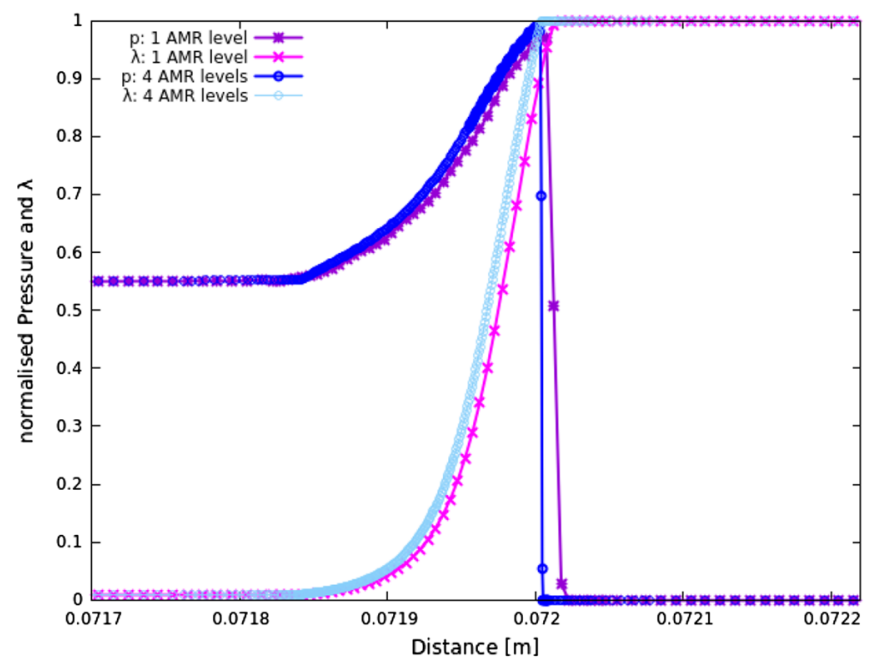

(c)

Fig. 1 Resolution study based on the convergence of the ZND detonation structure using five different resolutions. In a, the full ZND structure is shown in normalised (by the von Neumann value) pressure.
In $\mathbf{b}$, a magnified section of $\mathbf{a}$ illustrating the reaction zone is given. In c, the reaction rate variable is linked to the ZND structure to show the resolution of the reaction zone
Neumann peak values. To this end, a one-dimensional slab of liquid nitromethane is shocked to $23 \mathrm{GPa}$, a value close to the von Neumann pressure.

Following an initial unsteady evolution, the simulation settles to a steady detonation wave, yielding a constant value of the von Neumann spike pressure of $22.7 \mathrm{GPa}$ and a pressure at the $\mathrm{CJ}$ point equal to $12.6 \mathrm{GPa}$. These values fall within the range of values reported in the literature [25].

A resolution study was performed to demonstrate the convergence of the detonation wave for the resolutions considered in this work. A base resolution of $\mathrm{d} x=10 \mu \mathrm{m}$ is considered, and AMR levels with refinement factor 2 are added to refine the resolution. This leads to five effective resolutions considered in this section $\mathrm{d} x=5-0.3125 \mu \mathrm{m}$ corresponding to one up to five levels of AMR. In Fig. 1a, the steady-state detonation wave is shown in terms of normalised pressure (normalised by the von Neumann value), and in Fig. $1 \mathrm{~b}$ a zoom to the reaction zone is seen. Convergence for 2 and more AMR levels is seen. In Fig. 1c, the same pressure profiles for one and four AMR levels are shown, accompanied by the profile of $\lambda$ to demonstrate the number of points in the reaction zone. For the ZND test in this section, ignition tests in Sect. 3.2 and the neat nitromethane ignition of Sect. 4, a resolution of $\mathrm{d} x=0.625 \mu \mathrm{m}$ is used. This resolves well the reaction zone of liquid, neat nitromethane which is calculated from experiment [25] to have width of $\approx 300 \mu \mathrm{m}$. Operator splitting and subcycling were used for integrating the source term, leading to a timestep for the ODE comparable to the inverse of the pre-exponential factor in the Arrhenius law $\left(\mathrm{d} t_{\mathrm{ODE}}=1 / 2.6 \times 10^{9} \mathrm{~s}\right)$. 


\subsection{Comparison with experimental ignition times}

For the final part of this assessment, it is worth recalling that we are mainly interested in the early ignition stages of the shock-to-detonation process. It is not unusual to find that, depending on the mathematical formulation of the model, the reaction rate parameters used for detonation modelling are not suitable for ignition studies. This is attributed to the fact that the parameters are adjusted to fit post-shock temperatures and steady detonation values. To assess whether the current set-up can be employed for arbitrary studies without any further adjustment, we compare our numerical results against experimental Pop-plot data that show induction time (i.e., time to ignition) versus input pressure. The exact time when ignition occurs is problematic, since ideally, one would use the same definition of time of ignition for both numerical and experimental results. In numerical simulations, the time of ignition can be defined as the time when a specific fraction of the explosive material has reacted. The selection of the appropriate percentage should be based on experiments. However, experimentally it is rather difficult, where possible at all, to measure the chemical species, and ignition is usually measured using luminosity, something that is not trivial to accurately and consistently retrieve from the simulations. Thus, we arbitrarily define ignition to be the time when a small percentage (namely 10\%) of the explosive material has reacted and compare our numerical results against experimental data taken from Berke et al. [26], Hardesty [14], and Chaiken [27]. This comparison is presented in Fig. 2 and

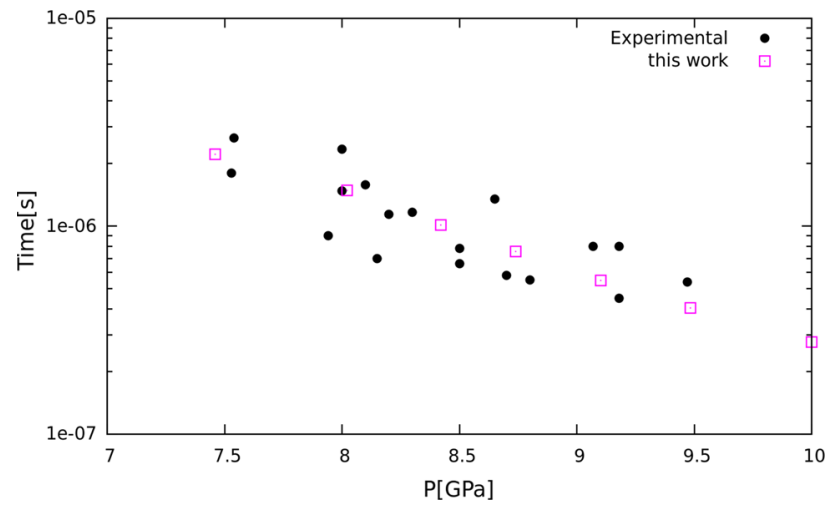

Fig. 2 Ignition time versus input pressure Pop plot. The black filled circles represent experimental data and the open squares numerically calculated data shows that the numerical results fall well within the range of the experimental ignition data.

\section{Ignition of neat nitromethane}

In condensed-phase explosives, initiation can be achieved by the passage of a shock wave through the quiescent explosive, raising the temperature and pressure and triggering the start of reaction.

To study the initiation process of nitromethane, simulations of the shock-induced ignition of neat, liquid nitromethane are performed.

Since the ignition process of this application is purely one dimensional, we consider a domain of dimensions $[0,3.2 \mathrm{~mm}]$ and effective grid size $\mathrm{d} x=0.625 \mu \mathrm{m}$. This resolution resolves well the steady-state reaction zone of liquid nitromethane which is calculated from experiment [25] to have a width of $\approx 300 \mu \mathrm{m}$. The initial conditions for this test are given in Table 2 .

When a shock wave is set up numerically as an initial condition, a start-up error is generated due to the symmetric Riemann problem [28]. This error has the form of a small well in the density distribution behind the shock wave, which translates into a small hill in the temperature field. Since the reaction rate we use to model the reactions in liquid nitromethane depends exponentially on the temperature, even a disturbance of a small magnitude in this field (here $\approx 20 \mathrm{~kg} \mathrm{~m}^{-3}$ ) would rapidly grow, generating a spurious hot spot. The hot spot would, in turn, lead to ignition earlier than it would be expected if the density field was clean. In order to remove the disturbance by extrapolating from the nondisturbed shocked state, or by cutting the domain at a point after the start-up error, the disturbance has to be sufficiently formed. Thus, before treating the error, the shock wave is allowed to travel some small but significant distance from its initial position. If reactions were turned on during this travel time, the numerical hot spot would affect the state behind the shock wave. To overcome this, an inert shock wave is allowed to travel the distance required for the start-up error to be adequately formed and then the part of the domain that is more than five cells behind the shock wave is cut off. After the cut-off, the simulation is restarted with the reactions turned on.

The cut-off of the domain is performed at time $0.02291 \mu \mathrm{s}$, and reaction is allowed to start at time $0.02291 \mu \mathrm{s}$. All the
Table 2 Initial conditions for the initiation of neat nitromethane

\begin{tabular}{lllllll}
\hline Region & $\rho_{1}\left(\mathrm{~kg} \mathrm{~m}^{-3}\right)$ & $\rho_{2}\left(\mathrm{~kg} \mathrm{~m}^{-3}\right)$ & $u\left(\mathrm{~m} \mathrm{~s}^{-1}\right)$ & $p(\mathrm{~Pa})$ & $\lambda$ & $z_{1}$ \\
\hline Shocked nitromethane & 1934 & 1934 & 2000 & $10.98 \times 10^{9}$ & 0 & $10^{-6}$ \\
Ambient nitromethane & 1134 & 1134 & 0 & $10^{5}$ & 1 & $10^{-6}$
\end{tabular}




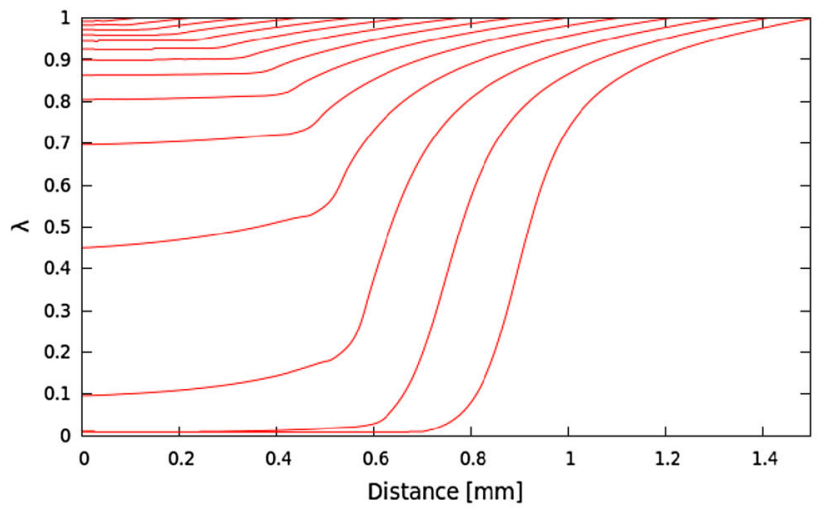

(a)

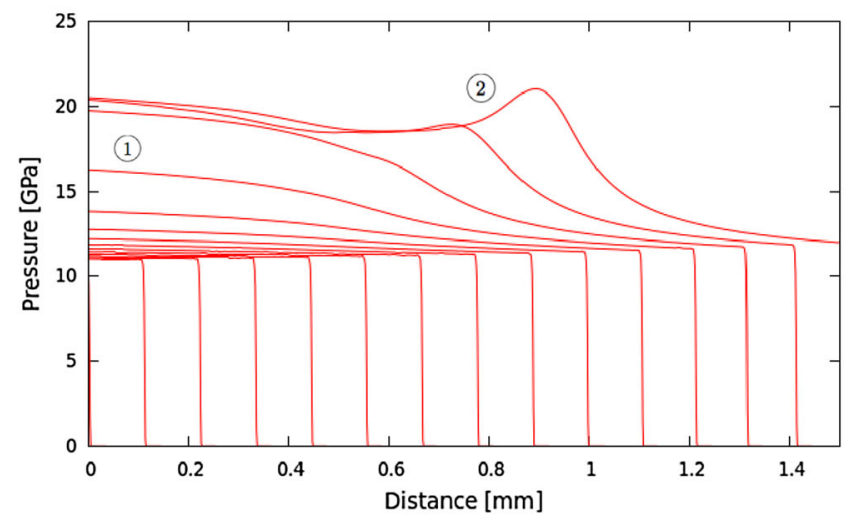

(c)

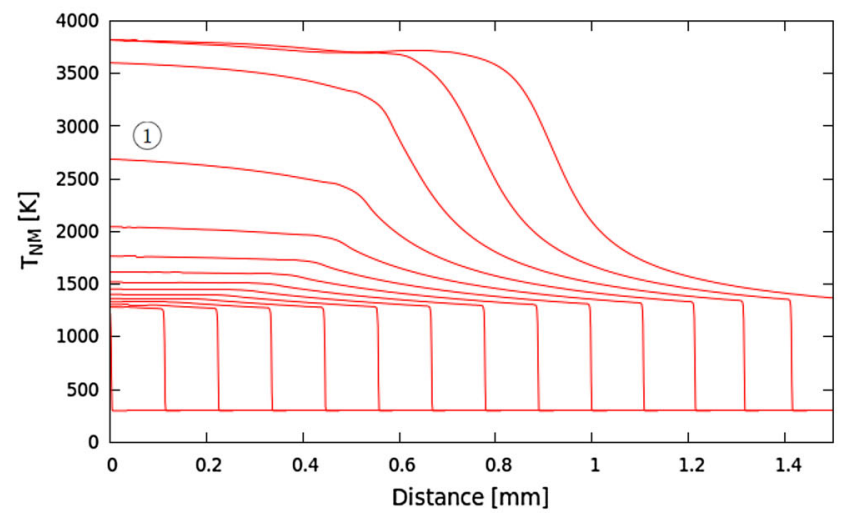

(e)

Fig. 3 One-dimensional evolution of the ignition and transition to detonation of neat nitromethane under the influence of a $10.98 \mathrm{GPa}$ shock wave at selected times. On the left, stages $0-14$ are illustrated and on

times referred hereafter are relative to the reaction start time. Also, all the positions are relative to the cut-off position $(x=$ $0.123 \mathrm{~mm}$ ), as the domain is considered to be repositioned at $x=0$ after the cut-off.

The evolution of the reaction progress variable $(\lambda)$, pressure $(p)$, and nitromethane temperature $\left(T_{\mathrm{NM}}\right)$ is illustrated in Fig. 3. As can be seen in the early stages of Fig. 3a, c, e, the fuel that is closer to the incident shock wave is shocked and

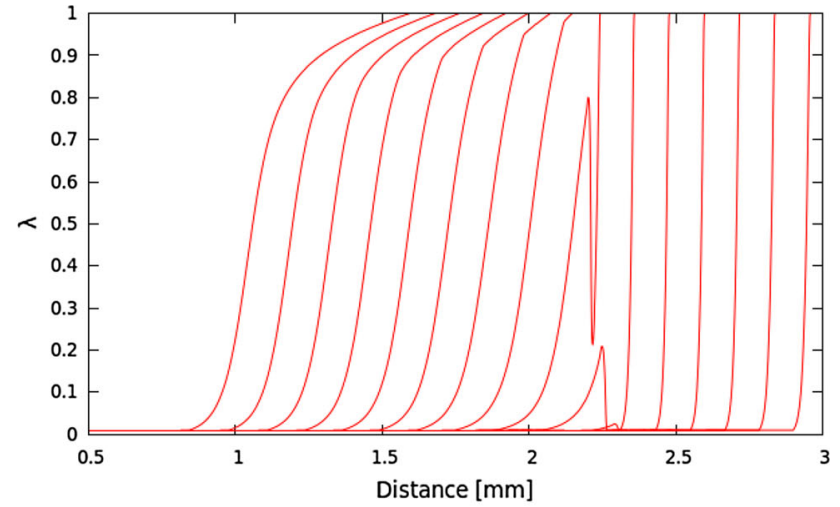

(b)

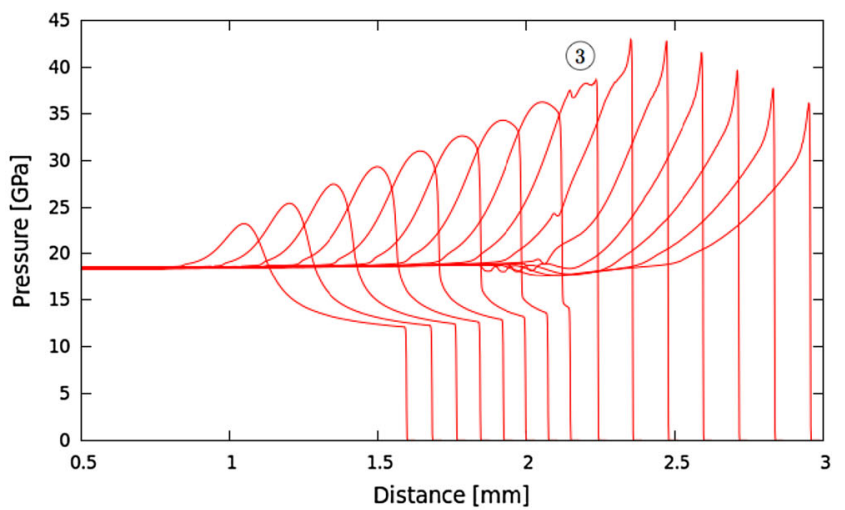

(d)

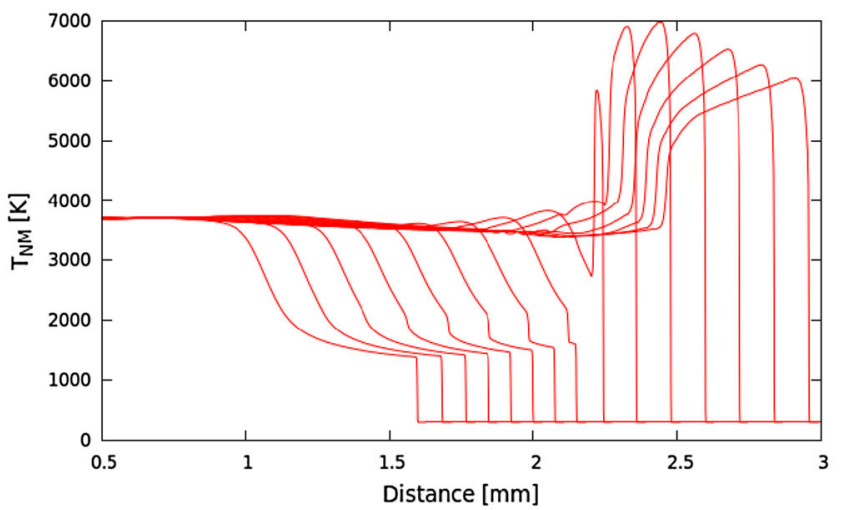

(f)

the right stages $15-29$. The top row illustrates the reaction progress variable $(\lambda)$, the middle row the pressure $(p)$, and the bottom row the nitromethane temperature $\left(T_{\mathrm{NM}}\right)$

heated first. Hence, it has more time to react than the fuel that is further away from the shock. As a result, temperature and mass-fraction gradients are generated (labelled (1)). During this process, explosive material is burnt and the reaction progress variable starts to deviate away from 1 . By defining as the ignition time the time when $\lambda=0.9$, we observe that here ignition occurs at stage 7 of Fig. $3 \mathrm{a}$ ( $\lambda$-plot), corresponding to $t_{\text {ign }}=0.16 \mu \mathrm{s}$. At the end of this slowly evolving induction 
Table 3 Initial conditions for the initiation of nitromethane by cavity collapse

\begin{tabular}{lllllll}
\hline Region & $\rho_{1}\left(\mathrm{~kg} \mathrm{~m}^{-3}\right)$ & $\rho_{2}\left(\mathrm{~kg} \mathrm{~m}^{-3}\right)$ & $u\left(\mathrm{~m} \mathrm{~s}^{-1}\right)$ & $v\left(\mathrm{~m} \mathrm{~s}^{-1}\right)$ & $p(\mathrm{~Pa})$ & $z_{1}$ \\
\hline Shocked nitromethane & 2.388 & 1934 & 2000 & 0 & $10.98 \times 10^{9}$ & 1 \\
Ambient nitromethane & 1.2 & 1134 & 0 & 0 & $10^{-6}$ \\
Air cavity & 1.2 & 1134 & 0 & 0 & $10^{5}$ & $10^{-6}$ \\
\hline
\end{tabular}

phase, the fluid cannot sustain the high pressure and temperature behind the shock wave, resulting in the generation of a signal, as seen during stages 13-14 of Fig. 3c (pressure plot, labelled (2). At this time $(t \approx 0.32 \mu \mathrm{s})$, thermal runaway is considered to occur. A rapid reaction stage follows the ignition stage, usually called the transition to detonation phase, during which the generated pulse is growing (Fig. 3d). At stage 23, the reaction wave overtakes the leading shock wave as shown by 3 in Fig. 3d. The overtake is accompanied by a rapid increase in pressure, temperature, and reaction (decrease of $\lambda$ ). Thereafter, the detonation structure settles down towards a steady-state solution. We also identify in this simple system of condensed-phase detonation the coherent coupling of pressure pulse with the heat release that results in the coupling between the reaction zone and leading shock wave. This is explained by the Shock Wave Amplification by Coherent Energy Release (SWACER) mechanism as described by Lee and Higgins [29].

\section{The collapse of a single cavity in reactive liquid nitromethane}

In this section, we consider an isolated gas-filled cavity of radius $0.08 \mathrm{~mm}$ collapsing in reacting liquid nitromethane in the domain spanning $[0,0.2 \mathrm{~mm}] \times[0.75,0.54 \mathrm{~mm}]$, with effective grid size $\mathrm{d} x=\mathrm{d} y=0.3125 \mu \mathrm{m}$. This grid size was determined by taking into account the resolution study in Part I for the collapse of the cavity in inert nitromethane as well as the convergence study for the detonation of reactive nitromethane described in Sect. 3.1. The initial conditions in the shocked, pre-shocked and cavity regions are given in Table 3. The domain is longer than in the inert simulations to allow for sufficient reaction to take place. It is also taller, to avoid the minor reflection of waves from the top boundary (even with transmissive boundaries) or the in-effect inflow that would occur if negative vertical velocities occur in the vicinity, both of which could affect the ignition process. We adopt the same abbreviations as in Part I.

The evolution of the reaction progress variable $\lambda$ (left), pressure $p$ (middle), and nitromethane temperature $T_{\mathrm{NM}}$ (right) is illustrated at selected times in Fig. 4.

The incident shock wave (ISW) travels within the nitromethane, compressing the material to $10.98 \mathrm{GPa}$ and raising the temperature to $1263 \mathrm{~K}$, as illustrated in Fig. 4a. In Part I of this work, we followed the generated wave pattern in detail and studied the effect of each wave on the temperature field. The wave patterns in this scenario are the same as those in Part I, and thus, we will not repeat the details of their generation. We will only describe the additional effects observed due to the presence of reactions.

At the initial stages of the collapse (see Fig. 4a, b) and away from the cavity (where the rarefaction wave (RW) from the collapse has not yet arrived), the reaction progress variable evolves as it would in a 1D neat nitromethane experiment. The expansion generated by the RW leads to a lowering of the temperature within the jet, and this affects the shape of the reaction zone. As a result, before the cavity collapses, the highest temperature in the nitromethane is in the uniform, unperturbed region behind the ISW and only minimal reaction is observed. After the cavity collapses (at $t=0.040 \mu \mathrm{s}$ ), we observe, for the first time in the collapse process, temperatures that are above the post-shock temperature. Specifically, the back collapse shock wave (BCSW) generates temperatures in the range $1263-3300 \mathrm{~K}$ (see Fig. 4c) accompanied by a small amount of reaction of $\lambda \approx 0.97$. The front collapse shock wave (FCSW) generates temperatures within the range $1263-1670 \mathrm{~K}$ (see Fig. 4c), in a region of almost no reaction at all $(\lambda \approx 0.99)$. The higher temperatures and the faster reaction at the rear of the cavity occur by the re-compression of the material that had already been shocked and was therefore preheated.

Ignition (in at least one reaction site) is observed at time $t_{\text {ign }}=0.0451 \mu \mathrm{s}$ in the back hot spot (BHS). The maximum temperature in the front hot spot (FHS) reaches at this point 2068 and $3089 \mathrm{~K}$ at the BHS.

For this set-up, the ISW proved slower than the nitromethane jet. Hence, at the time of collapse, the ISW is still traversing around the lobes. As a result of passing over the upper part of the upper lobe (and equivalently the lower part of the bottom lobe), many transmission/reflection processes take place. These processes result in a coalescence of waves (LSW) that are transmitted from the lobes into the pre-shocked (by the ISW) nitromethane residing around the lobes (Fig. 4d). This leads to the generation of temperatures higher than the post-shock temperature in the regions around the lobes. However, this temperature rise is not sufficient to generate a new ignition site in these timescales. 
(a)

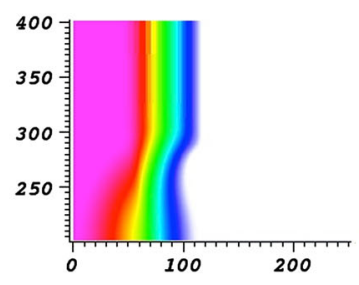

(b)

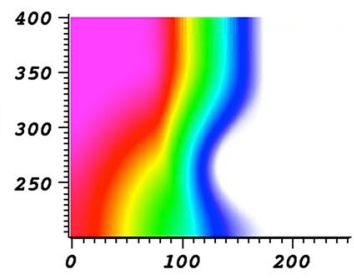

(c)

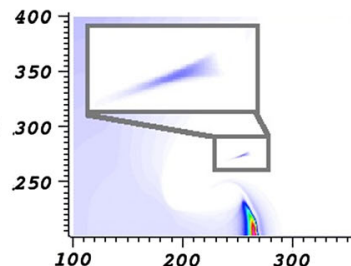

(d)

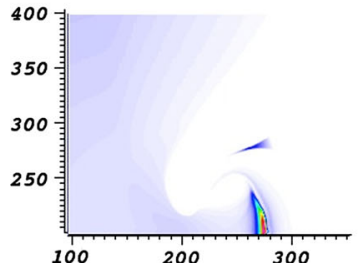

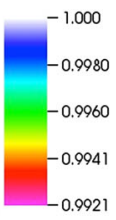
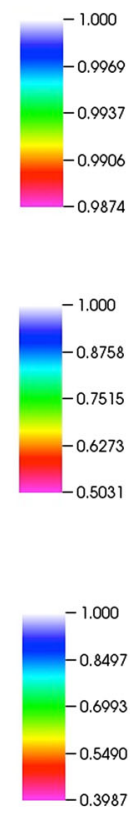
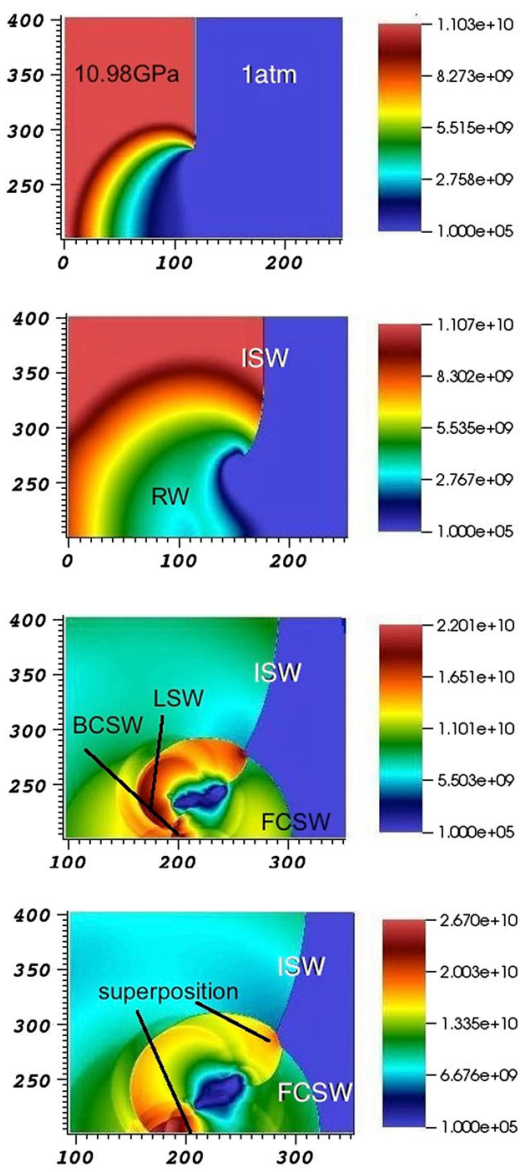
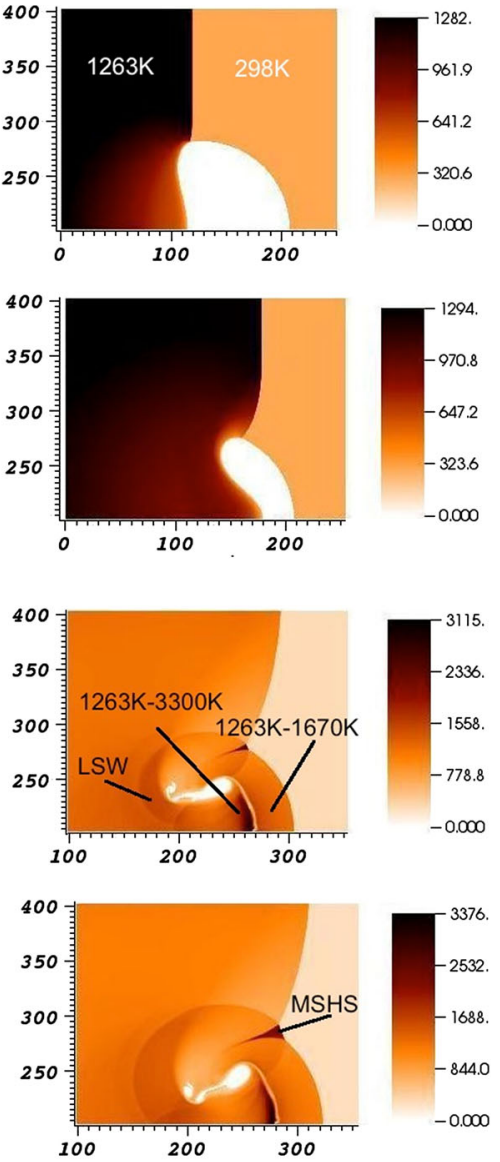

Fig. 4 Ignition process in the vicinity of a cavity embedded in liquid nitromethane following the passage of a $10.98 \mathrm{GPa}$ shock wave. The evolution of mass fraction $\lambda$ (left), pressure $p$ (middle), and nitromethane temperature $T_{\mathrm{NM}}$ (right) at times $t=\mathbf{a} 0.0229 \mu \mathrm{s}$, b $0.0351 \mu \mathrm{s}, \mathbf{c} 0.0591 \mu \mathrm{s}$, and $\mathbf{d} 0.0629 \mu \mathrm{s}$ is presented. The horizontal and vertical axes are space ordinates in $\mu \mathrm{m}$
The superposition of the ISW and the FCSW generates the Mach stem hot spot (MSHS) as described in Part I (Fig. 4d), which in the reactive scenario encloses temperatures of the range $1263-1493 \mathrm{~K}$. At this point, the highest overall temperature is still observed in the BHS $(2773 \mathrm{~K})$. As the Mach stem region grows, the temperature it encloses increases rapidly, reaching values of the order of $2700 \mathrm{~K}$. Ignition is observed in the MSHS at $t=0.0630 \mu \mathrm{s}$.

To the rear of the cavity, the waves emanating from the top and bottom lobes are superposed. Parts of them are superposed with the BCSW as well (see Fig. 4d). This results in regions that have been shocked twice or even three times and hence leads to temperatures of the order of $1600 \mathrm{~K}$.

In the final stages of the collapse process, the remains of the cavity are advected downstream and more burning is observed in the reaction sites, with $\lambda$ reaching a value of 0.0745 in the BHS and a value of 0.265 in the MSHS by the end of the simulation.

In Fig. 5 (top), the percentage of burning given as the maximum value of $100 \times(1-\lambda)$ in the centreline hot spots (BHS and FHS) and in the MSHS is shown over time. As time 0 we denote the time of birth of the hot spots which is the ignition time in each location identified earlier. It can be seen that the burning in the centreline hot spots (CHS) follows a $\sqrt{x}$ graph, while the MSHS burning increases roughly linearly. In Fig. 5 (bottom), the rate of burning in the two hot spots is presented. It can be seen that the reactions in the CHS grow faster initially than the reactions in the MSHS although this is reverted at later times and the reactions in the MSHS grow faster than the reaction in the CHS.

\subsection{Evolution along constant latitude lines}

It is informative to consider the evolution of the flow field and its effect on the temperature along lines of constant latitude. First, we discuss events along $y=0.21 \mathrm{~mm}$. This provides insight into the hot spot generation at the rear of the cavity (BHS), the minimal reaction at the front of the cavity (FHS) and the temperature distribution close to the centreline of the cavity. The evolution of the reaction rate variable $\lambda$, pressure, 


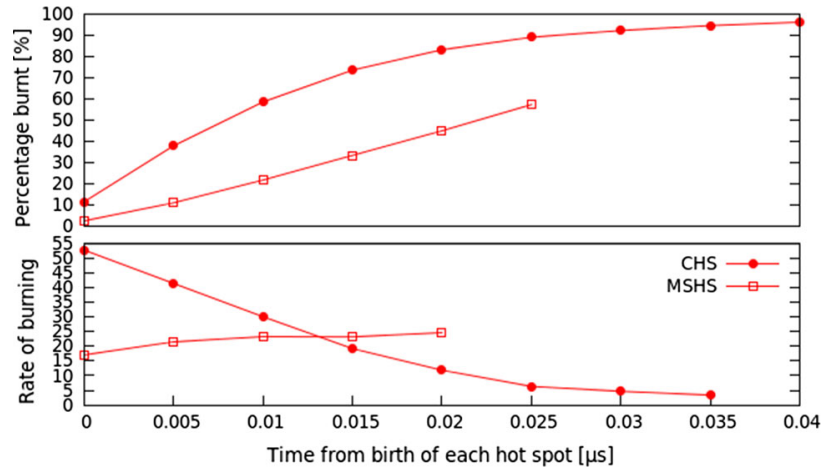

Fig. 5 Percentage of explosive burnt (top) and rate of burning (bottom) in the centreline hot spots and Mach stem hot spots from the time of their birth

and nitromethane temperature along this line is shown in Fig. 6.

At the early stages, a slight increase in the temperature field by the passage of the ISW is seen. Upon the interaction of the ISW with the cavity, a downstream travelling air shock and an upstream travelling RW are generated. The effect of the RW is a decrease in pressure and temperature. As it prop- agates, its effect becomes more profound, manifested as a further local decrease in the pressure and temperature fields and rate of reaction. The evolving crest-like feature (following the local decrease) seen in the pressure and temperature plots (Fig. 6a) is due to the formation of the nitromethane jet.

As the cavity collapses, the lineout crosses both shock waves (FCSW and BCSW), seen as two high-pressure and high-temperature fronts moving away from each other, labelled as (1), 2 in Fig. 6a. The BCSW compresses the material to a considerably higher pressure than the FCSW, generating a higher temperature at the rear of the cavity, compared to the front. Initially, the high-pressure fronts (i.e., BCSW and FCSW) coincide with the high-temperature fronts. However, as the collapse shock waves (CSWs) move away from the cavity, the pressure fronts (1), (2) propagate faster than the temperature fronts (3), (4)). The formation of the two CSWs leads to a considerable increase in the reaction rate, especially within the BHS (as indicated by the $\lambda$-plot in Fig. 6b). The reaction in the FHS is observed to be comparatively slow. Within the BHS and along this lineout, ignition is seen to occur at $t=0.0498 \mu \mathrm{s}$.
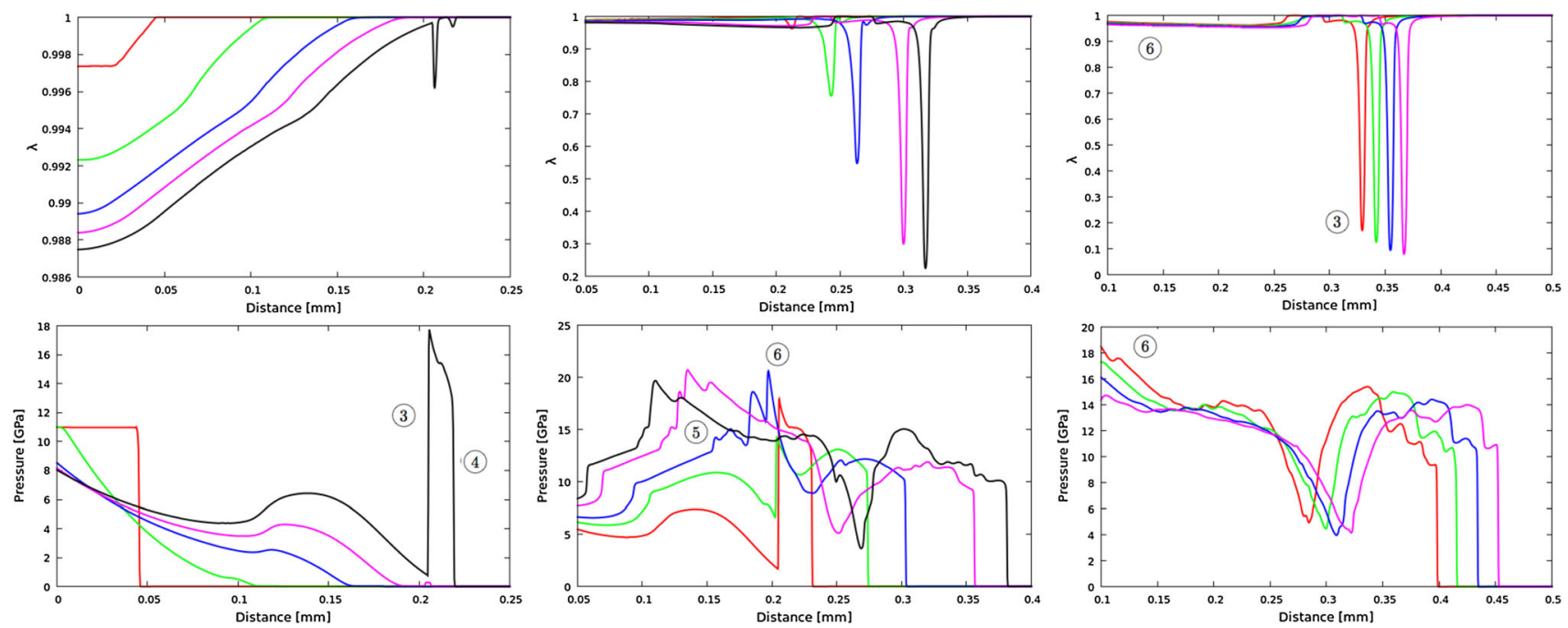

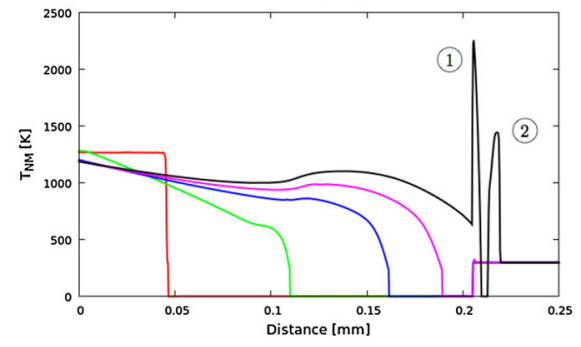

(a)

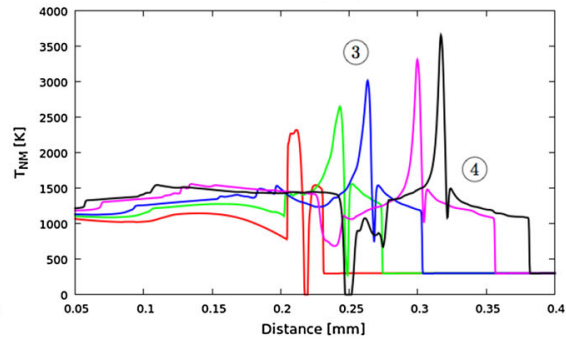

(b)

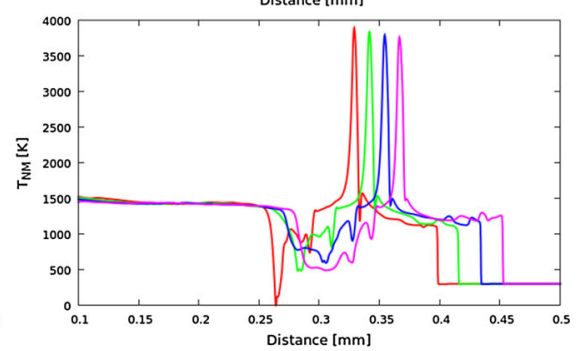

(c)
Fig. 6 Lineouts along $y=0.21 \mathrm{~mm}$ from Fig. 4. The mass fraction (top row), the pressure (middle row) and the nitromethane temperature (bottom row) are shown at stages a 4, 9, 14, 16, 18, b 19, 24, 27,
34,37 , c $38,41,43,45$, corresponding to times a $0.00892-0.0424 \mu \mathrm{s}$, b $0.0445-0.0780 \mu \mathrm{s}$, and c $0.0798-0.0932 \mu \mathrm{s}$ 

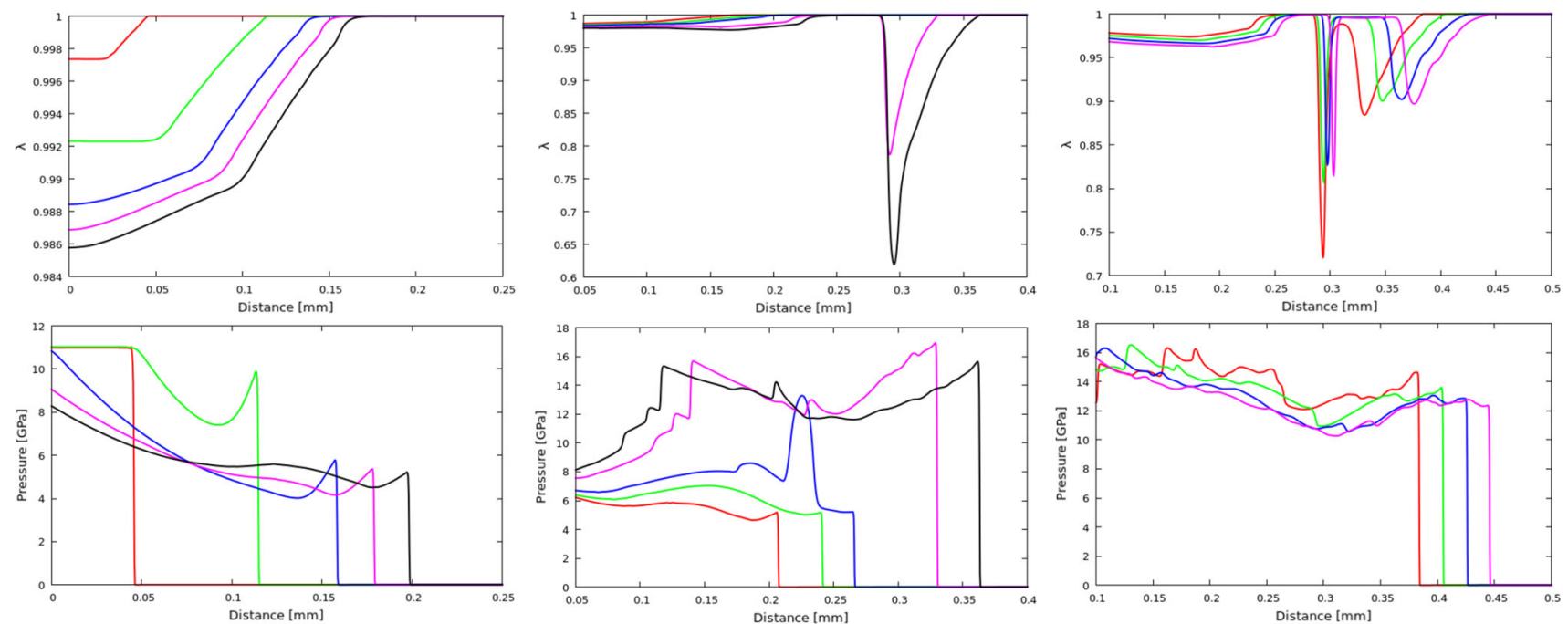

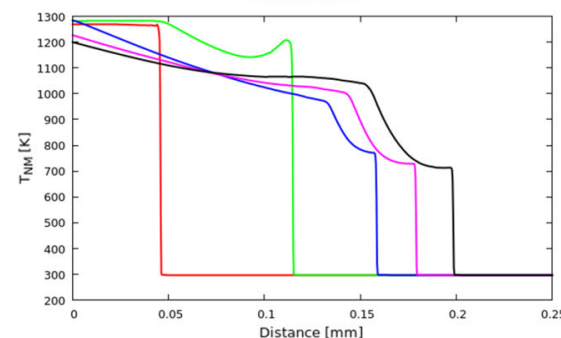

(a)

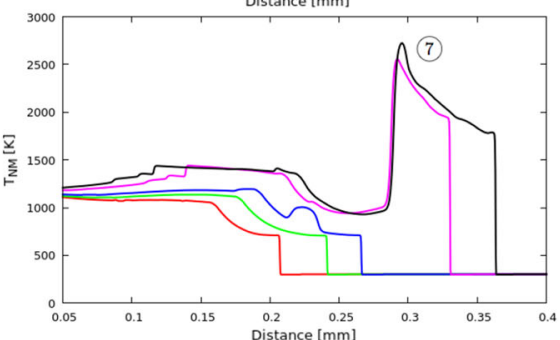

(b)

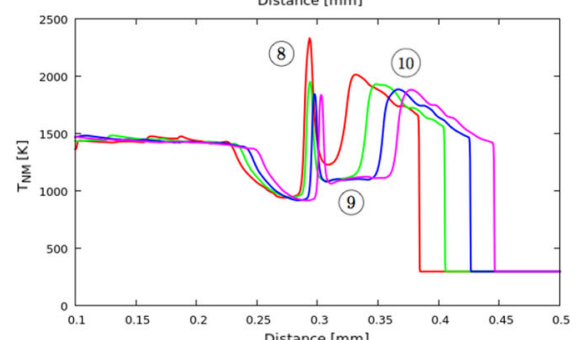

(c)
Fig. 7 Lineouts along $y=0.29 \mathrm{~mm}$ from Fig. 4. The mass fraction (top row), the pressure (middle row) and the nitromethane temperature (bottom row) are illustrated at stages a 4, 9, 14, 16, 18, b 19, 24, 27,
34,37 , c $38,41,43,45$, corresponding to times a $0.00892-0.0424 \mu \mathrm{s}$, b $0.0445-0.0780 \mu \mathrm{s}$, and c $0.0798-0.0932 \mu \mathrm{s}$
Waves emanate from the lobes after the collapse of the cavity and are labelled (5) as they intersect this line. Neither these nor their superposition with the BCSW results in a considerable increase in the temperature field. In fact, the highest temperature still occurs in the BHS. At stage 27, the upward wave from the lower lobe reaches the line $y=0.21 \mathrm{~mm}$. Part of this wave is superposed with both the BCSW and the wave entities emanating from the upper lobe, and part of it is superposed with the waves emanating from the upper lobe only. These superpositions increase the pressure locally but do not have a significant effect on the temperature. As these shock waves (labelled (6) move away from the cavity, the temperature and pressure along the line $y=0.21 \mathrm{~mm}$ do not increase further, but the reaction in the BHS still increases.

Focusing on the reaction progress variable field illustrated in the $\lambda$-plot of Fig. $6 \mathrm{c}$, it is observed that, at all late stages, the largest amount of reaction occurs in the BHS. Some reaction is also seen in the FHS and in the region that is traversed by the BCSW and the waves emanating from the lobes. After the ignition (stages 21-22), the fuel continues burning until it reaches the threshold of $\lambda=0.01$, where no more fuel is considered to be available for burning.
After stage 35, the advection of the rear remaining parts of the cavity reach the lineout, leading to the low pressure and temperature parts of the lineouts and the $\lambda=1$ plateau seen in Fig. 6c.

Lineouts at $y=0.29 \mathrm{~mm}$ are used to give insight about the MSHS and in general about the temperature distribution above the cavity. The evolution of $\lambda$, pressure and nitromethane temperature on this line is illustrated in Fig. 7.

At stage 26, the effect of the $S_{12,14,16}$ wave entity as described in Part I of this work is seen in the plots of Fig. $7 \mathrm{~b}$. The FCSW overtakes the ISW at stage 28, resulting in the increase in the temperature and the reaction. As the line $y=0.29 \mathrm{~mm}$ goes through a large part of the MSHS, the elevated temperature is not found in an isolated peak but is distributed within a region behind the moving Mach stem front (Fig. 7b, labelled as (7)). This elevated temperature region results in the increase in reaction, as illustrated by the $\lambda$ plot in Fig. $7 b$. From stages 44 onward, the temperature is first seen to increase in a hill-like form (labelled as (8), then decreases taking a well-like form (9) and then increases again taking the form of a hill, but with a shallower gradient than before (10). This is because at these late stages, the 

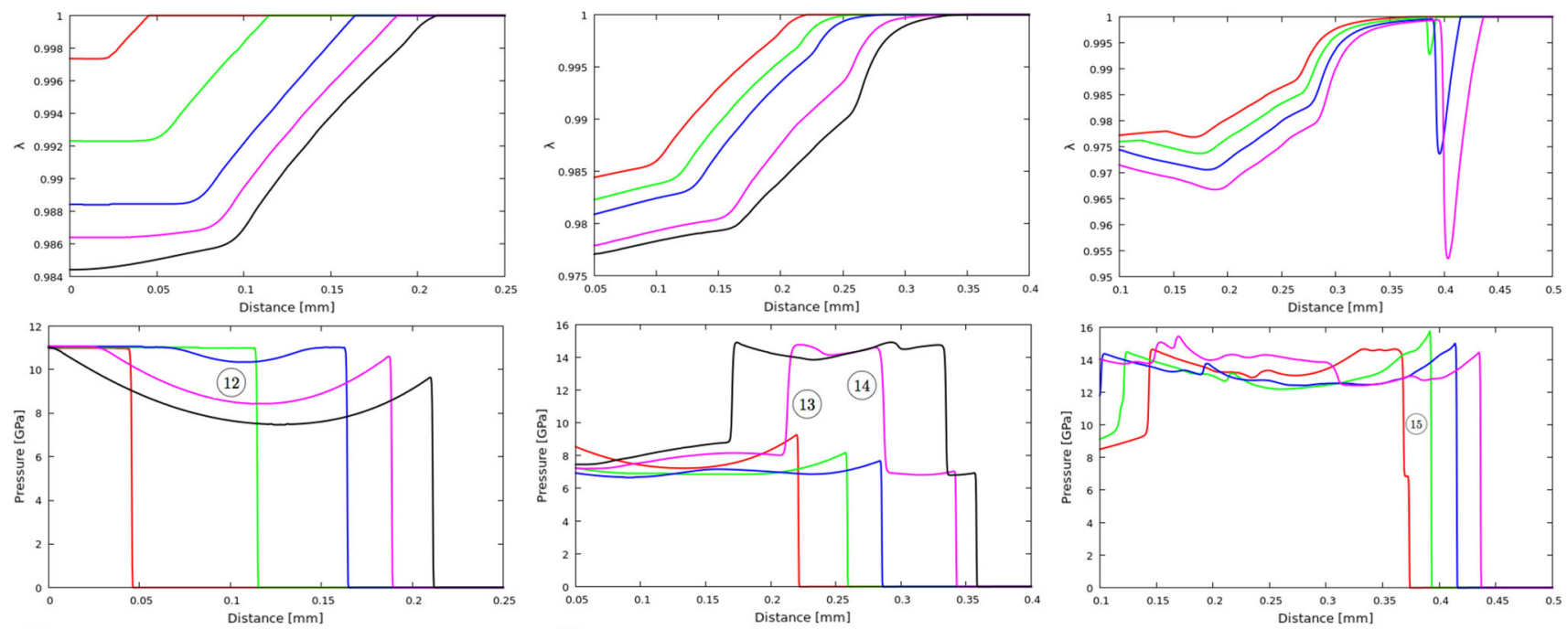

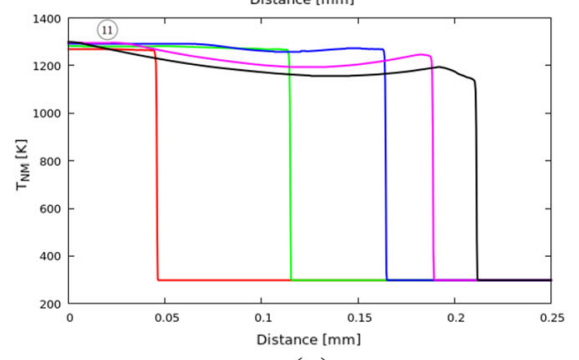

(a)

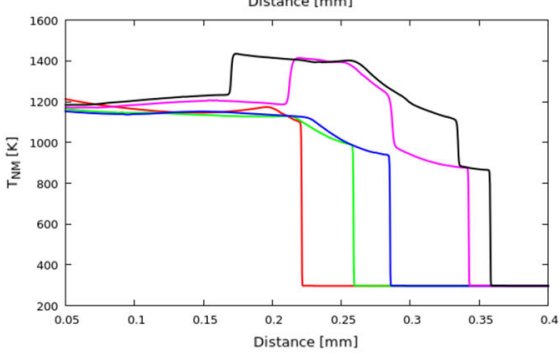

(b)

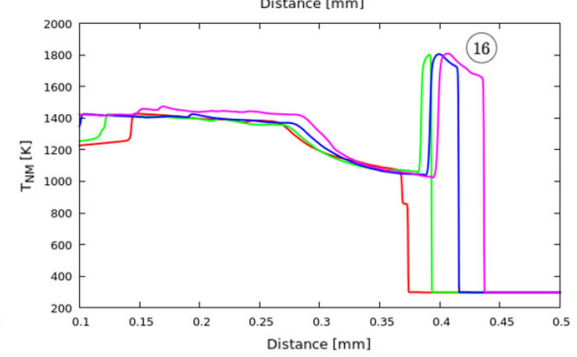

(c)
Fig. 8 Lineouts along $y=0.35 \mathrm{~mm}$ from Fig. 4. The mass fraction (top row), the pressure (middle row) and the nitromethane temperature (bottom row) are illustrated at stages a 4, 9, 14, 16, 18, b 19, 24, 27, 35,
37 , and c $38,41,43,44$, corresponding to times a $0.00892-0.0424 \mu \mathrm{s}$, b $0.0445-0.0780 \mu \mathrm{s}$, and c $0.0798-0.0932 \mu \mathrm{s}$
Mach stem feature has attained a horn-like shape and it is advected upwards. As a result, the line $y=0.29 \mathrm{~mm}$ intersects the stem, then goes through a region below the stem and then intersects the stem again. At the location of the second intersection, the temperature is elevated compared to the regions outside the stem, but it is lower than at the location of the first intersection. This translates as a well-hill-well feature in the $\lambda$-plot, indicating more reaction along the part of the line $y=0.29 \mathrm{~mm}$ that intersects the stem the first time, far less reaction outside the stem, and a moderate amount of reaction at the second intersection.

Another horizontal lineout, at $y=0.35 \mathrm{~mm}$, is used to give insight about the generation of the MSHS and temperature distribution and initiation around the cavity (Fig. 8). On this line, the effect of all the waves emanating from the cavity collapse process is seen later than on the previous lineouts considered.

In the early stages of Fig. 8a, a slight increase in the pressure and a more noticeable increase in $T_{\mathrm{NM}}$ due to the passage of the shock wave are seen (labelled as 11 in the temperature plot) resulting from the start of the reaction behind the shock wave in the $\lambda$-plot. The slight increase in the post- shock pressure, the formation of a temperature gradient and the shape of the $\lambda$-plot are as observed in the shock-induced ignition of neat nitromethane, because no effects from the collapse of the cavity have reached the line $y=0.35 \mathrm{~mm}$ yet.

By stage 14, the rarefaction wave (RW) has reached $y=$ $0.35 \mathrm{~mm}$ (as opposed to stages $8-9$ on $y=0.29 \mathrm{~mm}$ ) and its effect is seen as a descent in the pressure and temperature plot (labelled as 12 in the pressure plot). The effect of the RW is increased as the wave propagates upwards, seen as growth of the dip in pressure and temperature (Fig. 8b). In the $\lambda$-plot, the shape of the lower part of a graph is affected, presenting a slight increase of the gradient of the straight-line part of the graph. This indicates that less reaction is taking place when the rarefaction wave is present compared to the reaction that would have taken place in the absence of the rarefaction wave.

At stage 34 , the entity of waves $S_{12,14,16}$ is seen to have crossed the line $y=0.35 \mathrm{~mm}$ (as opposed to stage 26 for $y=0.29 \mathrm{~mm}$ ) and increases the pressure and temperature. As this wave is circular (spherical in 3D) and propagates outwards from the cavity, the area cut by the line increases 


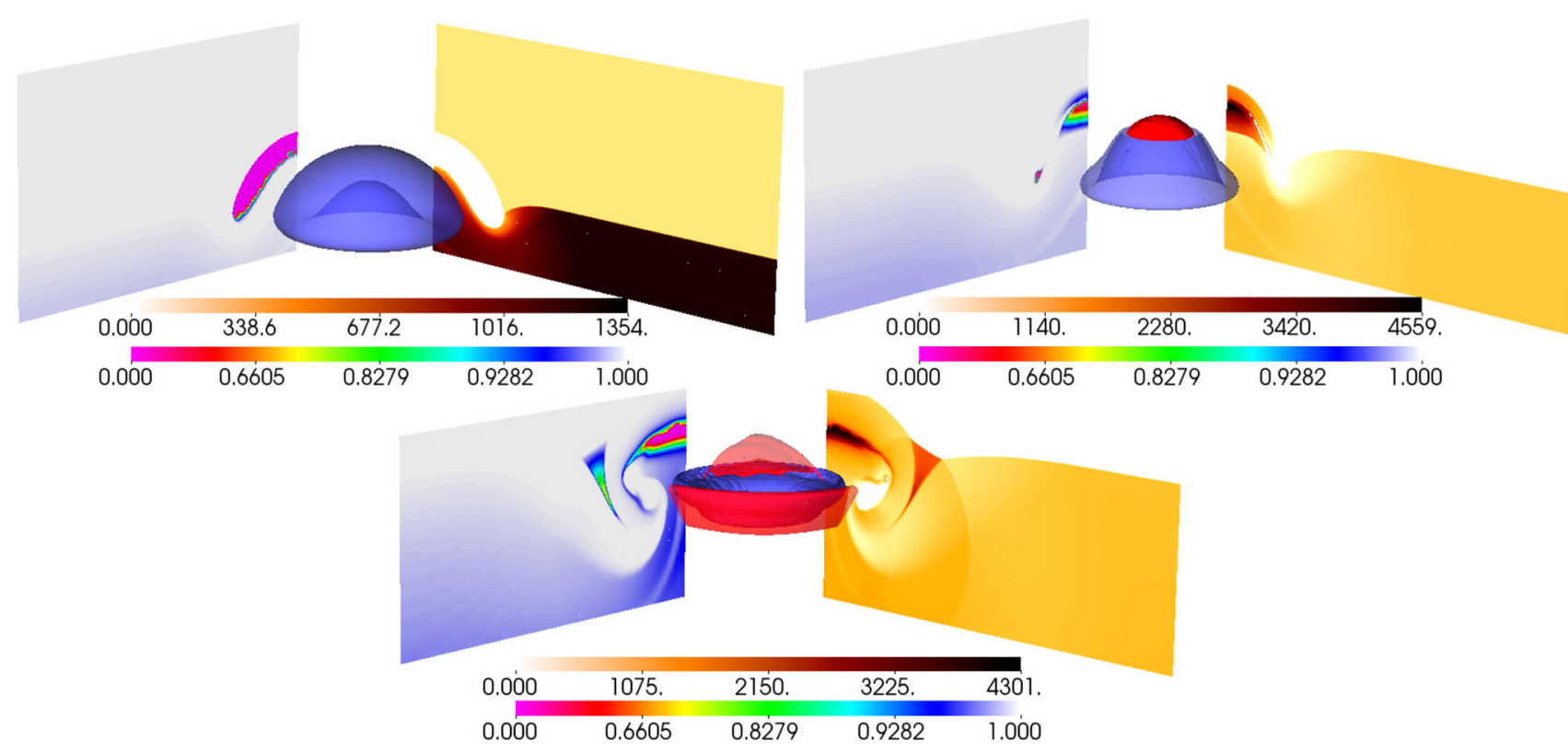

Fig. 9 Snapshots of the three-dimensional collapse of a cavity in reacting liquid nitromethane. The blue, three-dimensional $z_{1}=0.5$ contour represents the cavity boundary. The red, $\lambda=0.9$ contour represents the generated hot spot, i.e., the region where reaction takes place. A two-dimensional slice through the centre of the cavity is taken for the $\lambda$ and nitromethane temperature fields. The slices are projected on the

with time. As a result, in the one-dimensional pressure plot of Fig. 8b, this entity of waves is seen to be composed of an upstream travelling (labelled as 13 ) and a downstream travelling (labelled as 14 ) pressure wave. The effect of the wave entity $S_{12,14,16}$ (as seen in Part I) on the one-dimensional temperature field is seen in Fig. $8 \mathrm{~b}$ as an increase in $T_{\mathrm{NM}}$, in the form of two temperature fronts. Its effect on the reaction progress variable is seen in Fig. 8b. It appears as a dip in $\lambda$ attributed to the backward moving part of the wave, as a sudden but not very distinct drop in $\lambda$ attributed to the front part of the wave and as an overall decrease in the gradient of the straight-line part of the graph. All these features of the $\lambda$-plot indicate rapid increase in reaction as soon as the new shock waves re-shock the area.

Stage 38 (Fig. 8c) corresponds to the time before the FCSW overtakes the ISW on line $y=0.35 \mathrm{~mm}$ (as opposed to stage 28 for $y=0.29 \mathrm{~mm}$ ) and stage 39 to the time immediately after the overtake ${ }^{1}$ (labelled as 15 in the pressure plot). After the overtake, the lineout goes through the hightemperature Mach stem region, leading to the generation of the temperature peaks (labelled 16) and an increase in the reaction, as indicated by the sudden decrease in the reaction progress variable in the $\lambda$-plot. The temperature of the hot

\footnotetext{
${ }^{1}$ Overtake in this context refers to the overtake of the ISW by the CSW and not the overtake of the ISW by a detonation wave.
}

left half ( $\lambda$ field) and right half (nitromethane temperature field) of each figure. Note that the collapse process is shown here to move from bottom to top rather than left to right. The images here can be considered as rotated by $90^{\circ}$ counterclockwise compared to Fig. 4 . This is done solely for illustration purposes

spot in the Mach stem continues to gradually increase. This translates into the temperature peaks of Fig. $8 \mathrm{c}$, resulting in the increase in the reaction and the sudden drop in $\lambda$ in Fig. $8 \mathrm{c}$.

\section{Comparing the three-dimensional and two-dimensional cavity collapse in reacting nitromethane}

In this section, the three-dimensional (3D) collapse of a cavity in reacting nitromethane is presented and compared to the two-dimensional (2D) equivalent simulation presented in the previous section, with effective grid size $\mathrm{d} x=\mathrm{d} y=$ $\mathrm{d} z=0.3125 \mu \mathrm{m}$. Selected stages of the collapse are shown in Fig. 9. The three-dimensional $z_{1}=0.5$ contour represents the cavity material boundary, while the $\lambda=0.9$ contour represents the generated hot $\operatorname{spot}(\mathrm{s})$, i.e., the region where reaction takes place. We take a two-dimensional slice of the $\lambda$ field through the centre of the cavity and project it on the left half of each figure. This again illustrates the reaction regions. Similarly, the projection of the nitromethane temperature field on the same plane is seen on the right half of each figure. It is therefore presented how the generation of locally high temperatures leads to the generation of hot spots. The highest temperatures are located in front and behind the point of collapse of the cavity (FHS and BHS) as well as in the 


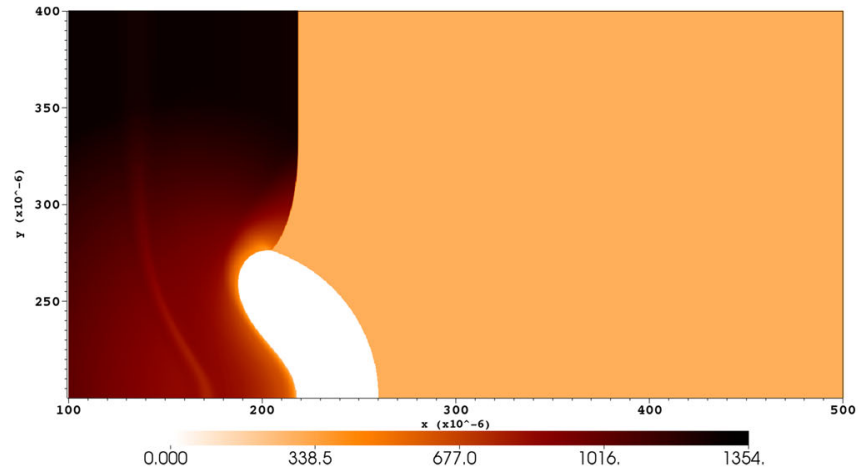

(a)

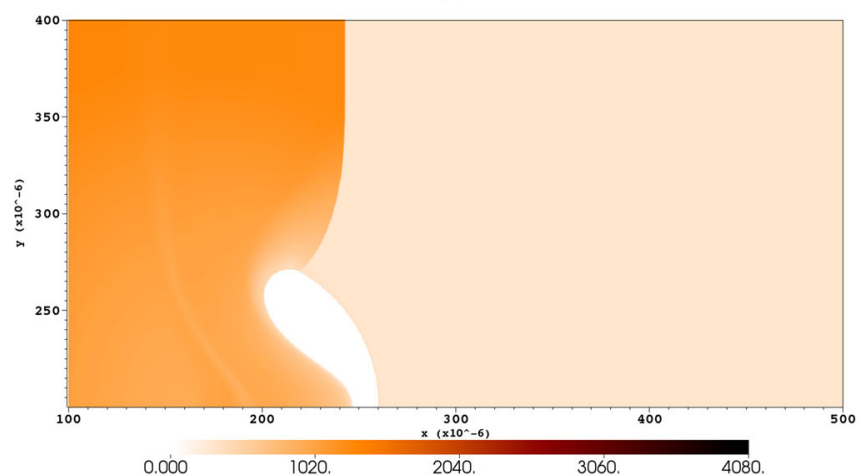

(c)

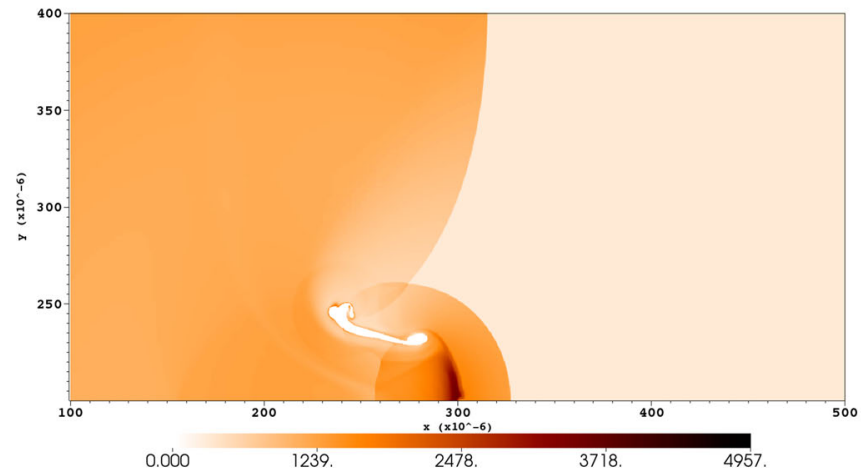

(e)

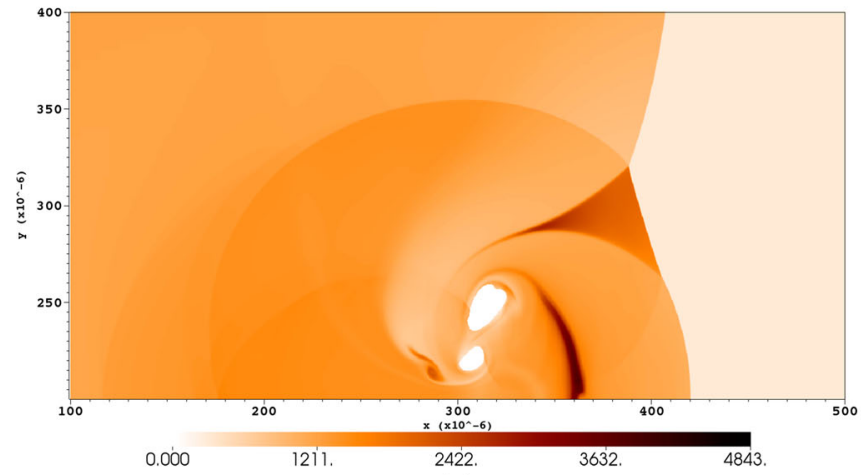

(g)

Fig. 10 Comparison of the temperature field and collapse times between a two-dimensional and a three-dimensional simulation of the cavity collapse under a $10.98 \mathrm{GPa}$ ISW at selected times. a 2D

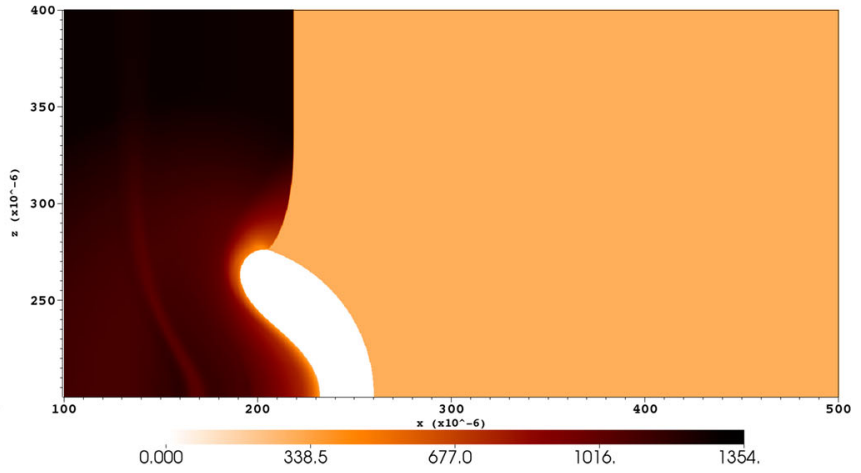

(b)

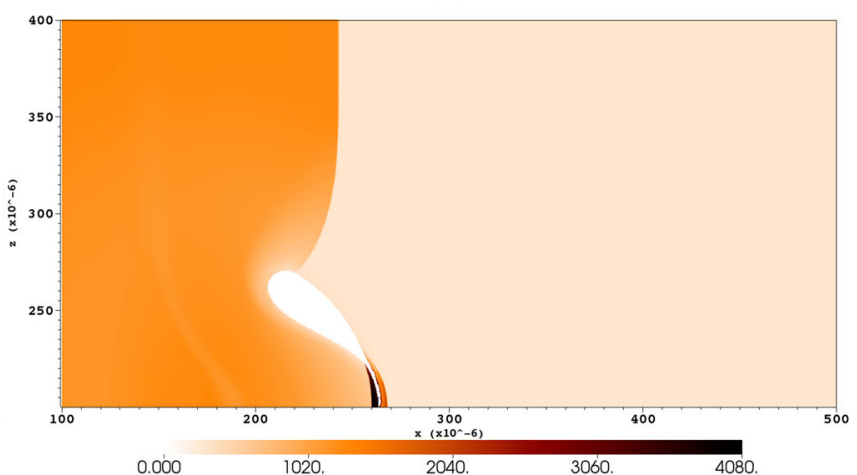

(d)

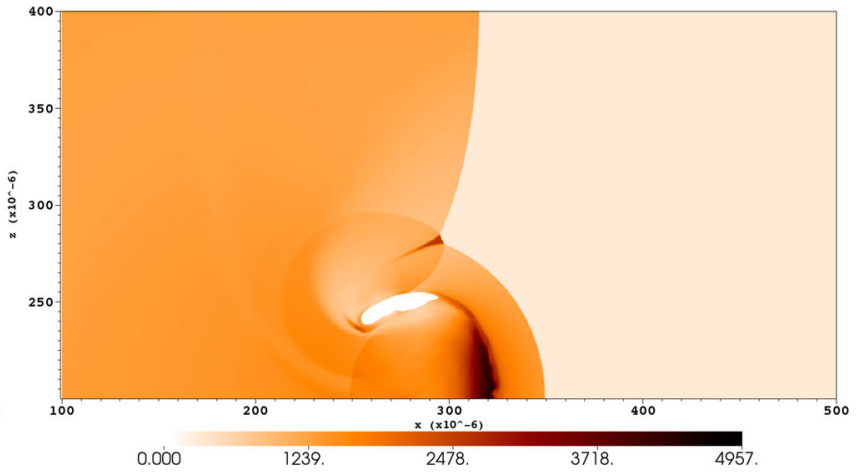

(f)

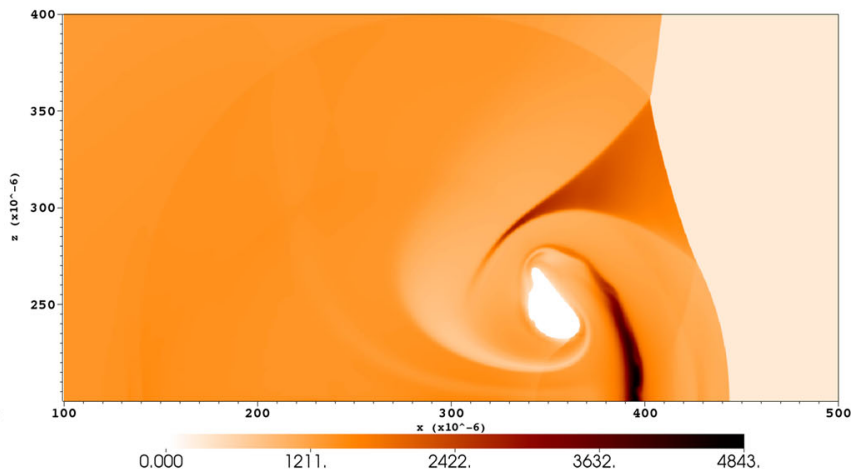

(h)

$t=0.03 \mu \mathrm{s}, \mathbf{b} 3 \mathrm{D} t=0.03 \mu \mathrm{s}, \mathbf{c} 2 \mathrm{D} t=0.035 \mu \mathrm{s}, \mathbf{d} 3 \mathrm{D} t=0.035 \mu \mathrm{s}$, e $2 \mathrm{D} t=0.05 \mu \mathrm{s}, \mathbf{f} 3 \mathrm{D} t=0.05 \mu \mathrm{s}, \mathbf{g} 2 \mathrm{D} t=0.07 \mu \mathrm{s}, \mathbf{h} 3 \mathrm{D} t=0.07 \mu \mathrm{s}$ 


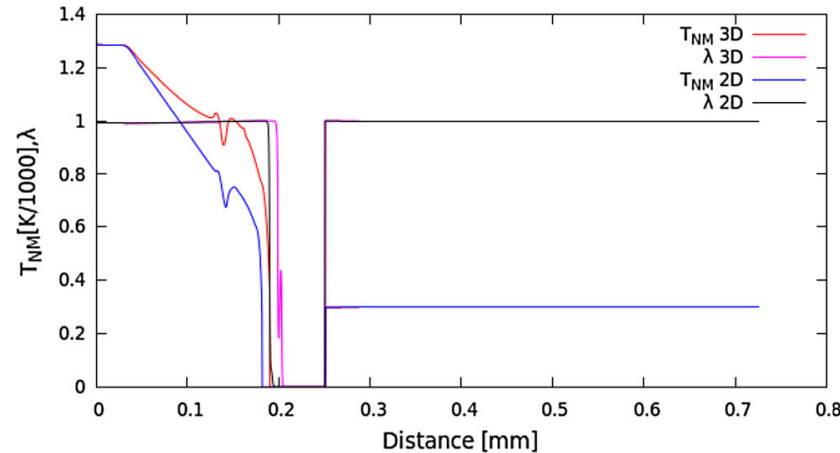

(a)

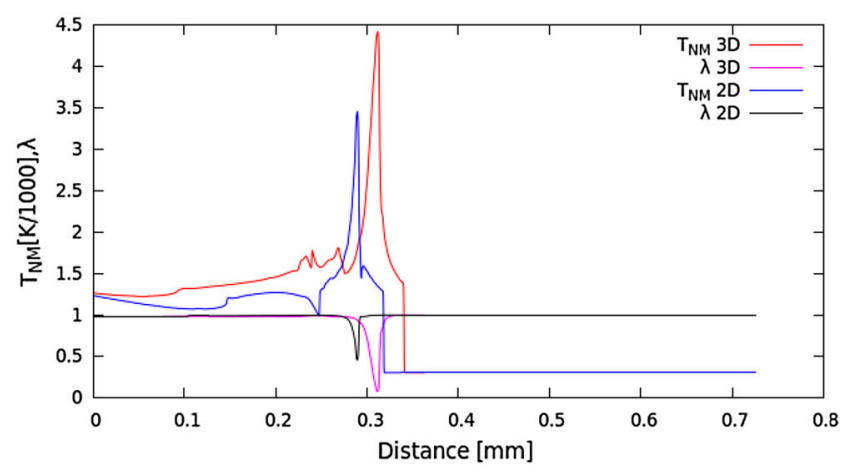

(c)

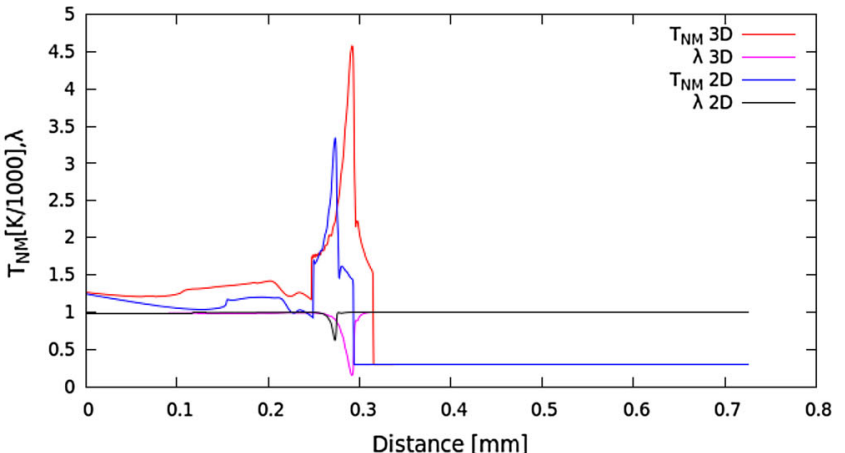

(b)

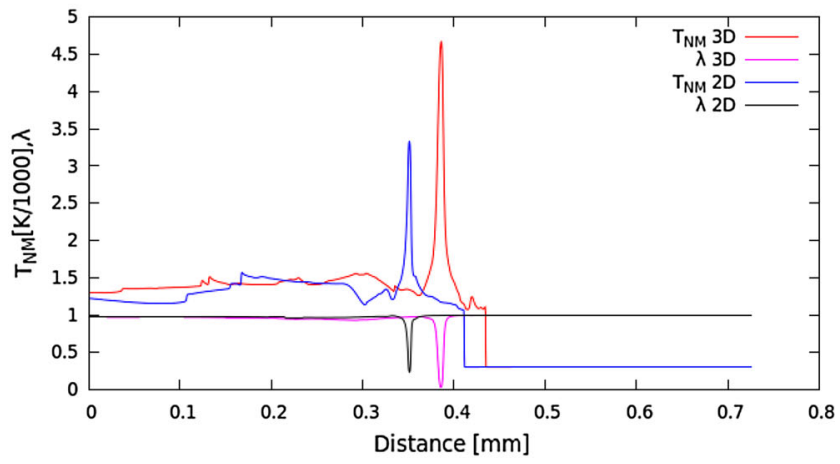

(d)

Fig. 11 Comparison of the temperature field and reaction along $y=0.21 \mathrm{~mm}$ in the $2 \mathrm{D}$ and $3 \mathrm{D}$ configurations. $\mathbf{a} t=0.025 \mu \mathrm{s}, \mathbf{b} t=0.045 \mu \mathrm{s}$, $\mathbf{c} t=0.05 \mu \mathrm{s}, \mathbf{d} t=0.07 \mu \mathrm{s}$

Mach stem generated at late stages of the collapse due to wave superposition. These lead to the generation of bell-shaped hot spots, mainly behind the point of collapse (BHS) and a torus-shaped hot spot corresponding to the three-dimensional Mach stem. The comparison between the 3D and 2D collapse process is performed by using planar pseudo-colour plots of the temperature field (Fig. 10) and the evolution of the temperature and $\lambda$ fields along lines of constant latitude, specifically along the centreline of the cavity and $y=29 \mu \mathrm{m}$ (Figs. 11, 12).

The three-dimensional nature of the flow field around the cavity results in a faster jet compared to the two-dimensional equivalent scenario. This is seen in Figs. 10a, b and 11a and effectively results in a faster nitromethane jet (1.4 times faster) in the $3 \mathrm{D}$ case than in the $2 \mathrm{D}$ case and the earlier observation of all subsequent features of the collapse phenomenon. The cavity collapses, as a result, faster by $\approx 0.5 \mu \mathrm{s}$ in the 3D case (Fig. 10c, d) and the generated collapse shock waves lead to a quicker ignition in the front and back hot spots (Fig. 11b). The temperatures achieved upon collapse range between 400 and $1500 \mathrm{~K}$ higher in the 3D scenario than in the 2D. The percentage of explosive burnt in the centreline hot spots in the centreline hot spot region is shown as a function of time in Fig. 13. It can be seen that the higher temperature attained upon the 3D collapse generates a higher immediate burn of the material in 3D (59\% burnt) compared to $2 \mathrm{D}$ (11\% burnt) and a faster overall ignition and burning procedure. The Mach stem and hence the MSHS are also generated quicker than in the 2D case (Figs. 10e, f, 12b). The Mach stem and the MSHS grow faster in the 3D case as well, as seen in Figs. 10g, h and 12c, d. In general, in the 3D case, higher temperatures are also achieved compared to the $2 \mathrm{D}$ case, as it is evident in Figs. 11c, $\mathrm{d}$ and 12c, d leading to faster immediate ignition.

\section{Comparing the cavity collapse in inert and reacting nitromethane}

In Part I of this work, the collapse of a cavity in non-reacting nitromethane was studied, and this work extended that to the equivalent reacting medium. In this section, the temperature field generated through the collapse process in the inert and reacting media is compared, to examine the effect of the reactions on the temperature field and the hot spot topology. The comparison uses the two-dimensional scenarios for simplicity. The number of hot spots generated in the two cases is identical, and the location is roughly the same; any difference observed is of the order of 3-4\% of the bubble radius and mainly in the BHS and FHS. The significant difference in the 


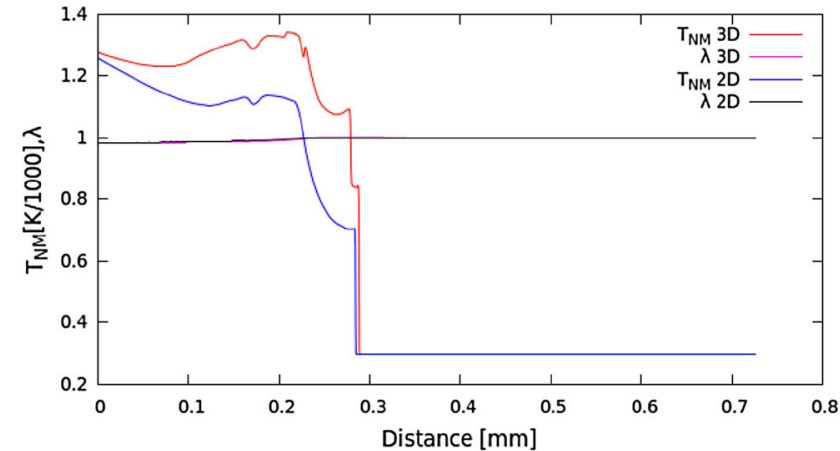

(a)

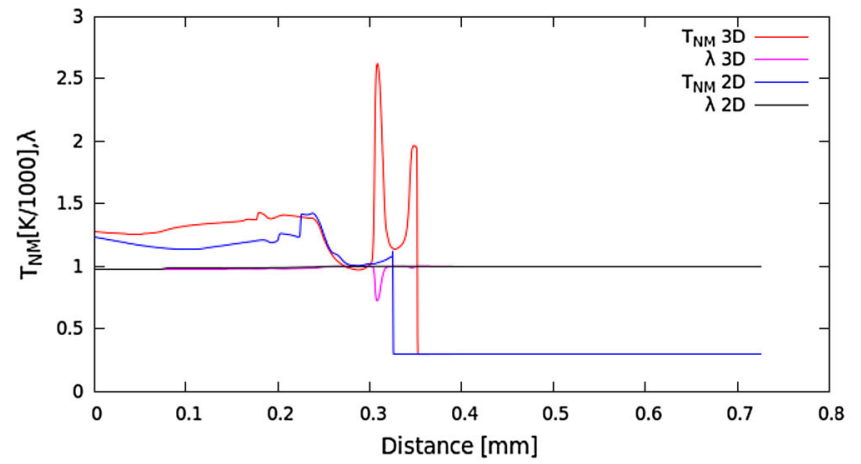

(c)

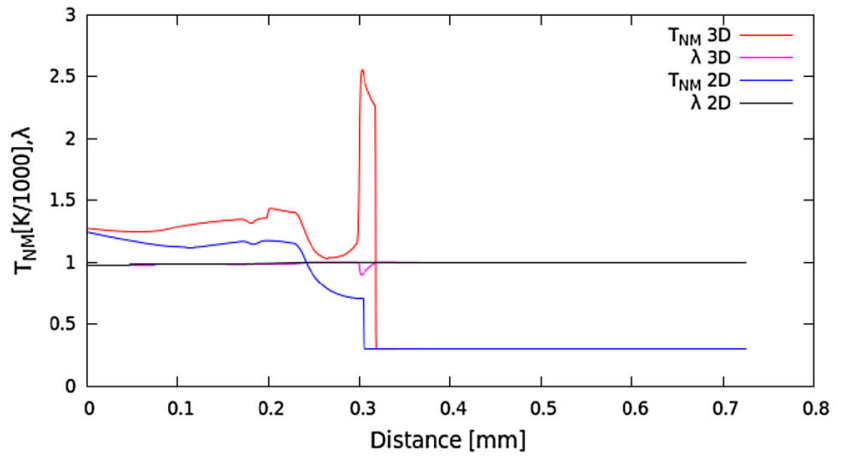

(b)

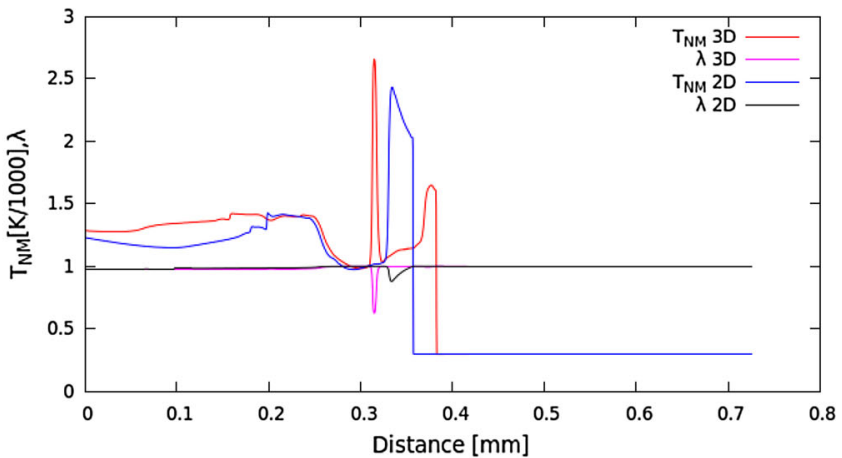

(d)

Fig. 12 Comparison of the temperature field and reaction along $y=0.29 \mathrm{~mm}$ in the $2 \mathrm{D}$ and $3 \mathrm{D}$ configurations. a $t=0.05 \mu \mathrm{s}, \mathbf{b} t=0.055 \mu \mathrm{s}$, c $t=0.06 \mu \mathrm{s}, \mathbf{d} t=0.065 \mu \mathrm{s}$

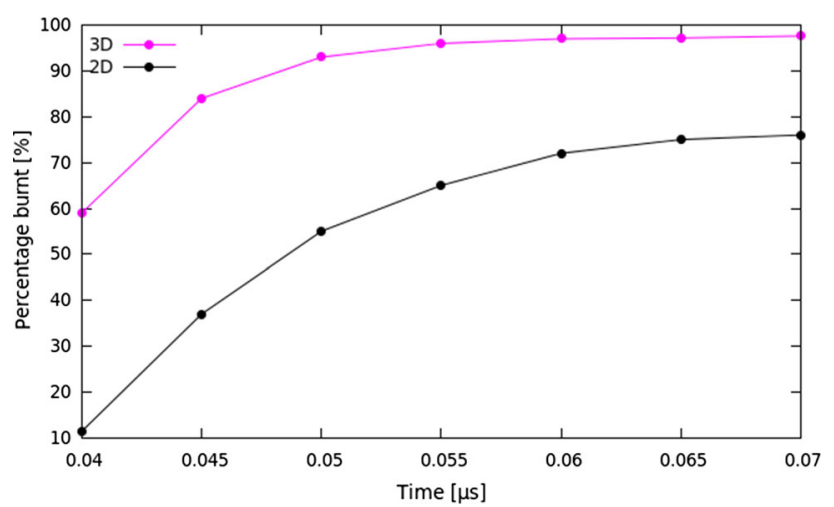

Fig. 13 Percentage of explosive burnt over time in the threedimensional and two-dimensional scenarios

two collapse cases is seen in the temperature field. At the early stages of the simulation, before the collapse takes place, some initial reaction is observed behind the shock wave, which is translated to some increase in the temperature field in the reactive case, compared to the inert case. The difference in the temperature fields of the two cases is small, ranging from 0 to $50 \mathrm{~K}$. Once the cavity collapses, the BCSW and FCSW generate significant reaction in the BHS and FHS leading to an even larger increase in temperature in the reactive case and a difference of $65 \mathrm{~K}$ from the inert case. This difference grows as the reaction progresses and the hot spots grow in size and strength. This is shown in Fig. 14, where the maximum temperature in the centreline hot spots (CHS), i.e., BHS and FHS, and in the MSHS is shown. The general trend is that after the collapse, the maximum temperature observed in the simulation in the reactive case increases, whereas it decreases in the inert scenario. This is expected as the reaction supports the shock waves and vice versa. In the reactive case, the maximum temperature observed can be higher than in the inert scenario by as much as $1500 \mathrm{~K}$. For this configuration and the timescales studied, the temperatures in the MSHS are always lower than the CHS. Moreover, the difference between the CHS and MSHS is larger in the reactive case than in the inert case.

The comparison of the rate of increase in the temperature in the CHS and MSHS between the inert and reactive scenarios is presented in Fig. 15. It is clear that the rate of increase in the maximum temperature in both hot spots is positive in the reactive case, i.e., the maximum temperature is always increasing, whereas this is not true for the inert scenario. The first peak in both loci corresponds to the birth of the hot spots. In the CHS a second, late peak is observed which corresponds to the superposition of the waves emanating from the two lobes along the centreline of the cavity. In the MSHS, the increase in the maximum temperature relies purely on 


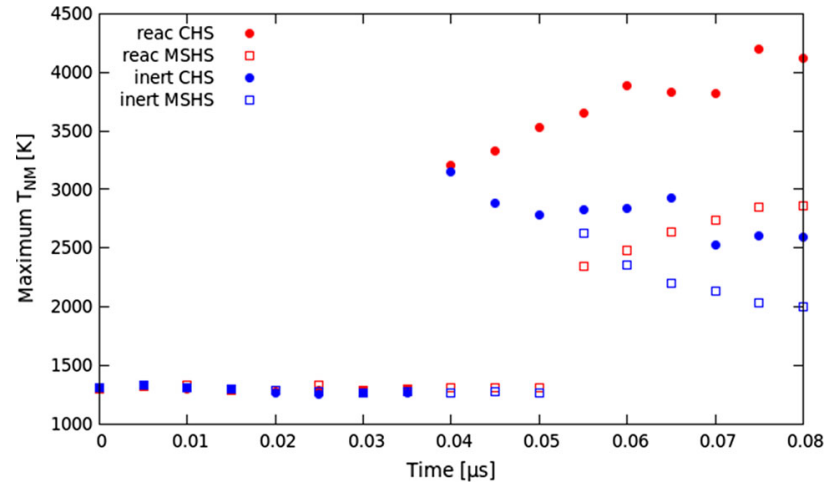

Fig. 14 Maximum nitromethane temperature observed in the centreline hot spots (BHS and FHS, labelled collectively as CHS) plotted using circles and in the MSHS plotted using squares. The red colour denotes results from the reactive set-up and the blue colour from the inert set-up

the waves generating the Mach stem. We also observe that the rate of increase in the MSHS in the inert case is always higher than the CHS (except at the birth of the hot spot). This is, however, not true for the reactive case. Comparing information from Figs. 14 and 15, we can conclude that in maximum temperature terms and in this configuration, the CHS are stronger in absolute value than the MSHS, although the growth rate of reaction in the MSHS was seen to be higher than in the CHS at late stages of the collapse in Fig. 5.

Using lineouts, we look at the temperatures in the different hot spots. The local temperature difference is higher in the late stages after the collapse so we omit illustrations of the early times. From $t=0.045 \mu \mathrm{s}$, the temperature difference in the BHS is more significant than in the FHS, until the two hot spots merge ( $t=0.065 \mu \mathrm{s})$ in Fig. 16a. The temperature difference in the MSHS is also significant, as seen on $y=$ $0.29 \mathrm{~mm}$ in Fig. $16 \mathrm{~b}$ and on $y=0.35 \mathrm{~mm}$ in Fig. $16 \mathrm{c}$ for $t=0.07 \mu \mathrm{s}$. The width of the MSHS is also larger in the reactive case.

\section{Conclusions}

In this work, we perform resolved numerical simulations of cavity collapse in liquid nitromethane using a multi-phase formulation, which can recover reliable temperatures in the vicinity of the cavity. Considerable care is taken regarding the form of equations and numerical algorithm to eliminate spurious numerical oscillations in the temperature field. The model is validated against experimental data. Specifically, we demonstrate that the deduced CJ and von Neumann values match the values found in the literature and also that the experimentally determined ignition Pop-plot data are matched. Following the validation, we study the shockinduced cavity collapse in reacting liquid nitromethane, in

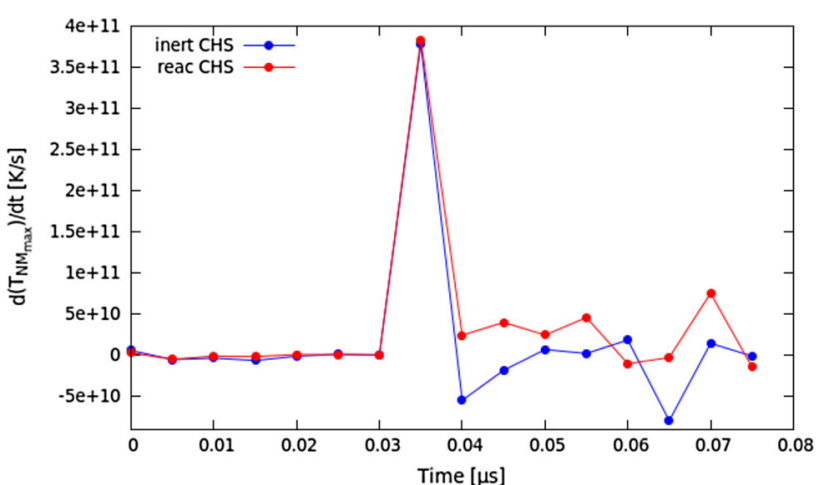

(a)

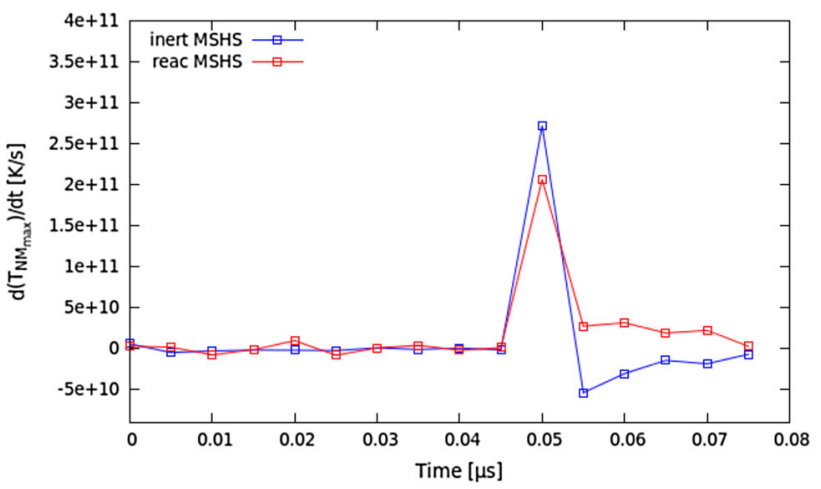

(b)

Fig. 15 Comparison of the rate of increase in maximum temperature $\mathbf{a}$ in the centreline hot spots (CHS) and $\mathbf{b}$ in the Mach stem hot spot (MSHS) in the inert and reactive scenarios

two and three dimensions, and follow the events leading to the generation of local temperatures and initiation of the explosive.

Working towards elucidating the relative contribution of fluid dynamics and chemical reaction, we examined in Part I of this work the details of the hydrodynamic effects that lead to local temperature elevations and identified a more complex hot spot topology than previously described in the literature. In this second part of the work, we demonstrate the effect of chemical reactions that acts additively to the fluid dynamics. We identify which high- temperature regions lead to reactive hot spots and observe much higher temperatures (40-1500 K) than in Part I.

We examine additionally the ignition of nitromethane in the absence of cavities and compare the ignition times for the neat and single-cavity material. We observe that the initiation of nitromethane in the presence of an isolated collapsing cavity is reduced to less than one-third (in 2D simulations or less than a quarter in $3 \mathrm{D}$ simulations) of the required time for igniting the neat material. This quantifies the sensitisation character of the cavities in this configuration.

It is observed that the highest nitromethane temperatures still occur in the back hot spot (BHS) as also observed in 


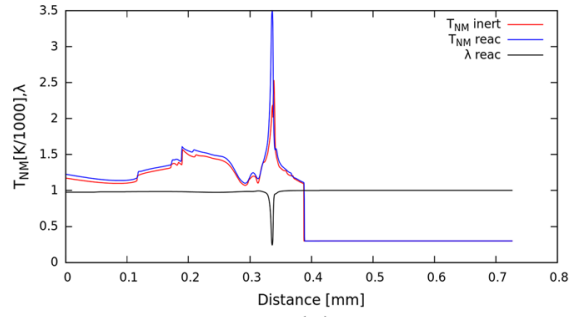

(a)

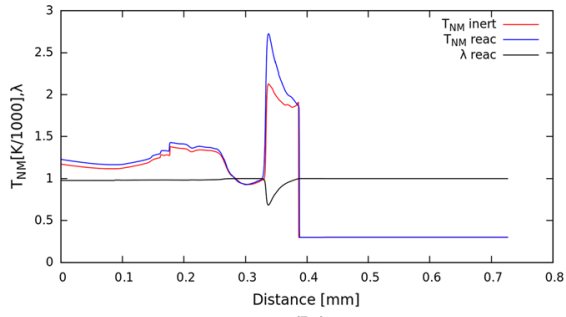

(b)

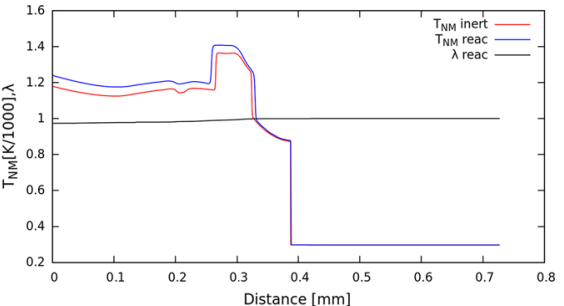

(c)

Fig. 16 Comparison of the temperature field and reaction along a $y=0.35 \mathrm{~mm}$ at $t=0.065 \mu \mathrm{s}, \mathbf{b} y=0.29 \mathrm{~mm}$, and $\mathbf{c} y=0.35 \mathrm{~mm}$ at $t=0.07 \mu \mathrm{s}$ between the inert and reactive configurations. a $y=0.29 \mathrm{~mm}, t=0.065 \mu \mathrm{s}, \mathbf{b} y=0.29 \mathrm{~mm}, t=0.07 \mu \mathrm{s}, \mathbf{c} y=0.35 \mathrm{~mm}, t=0.07 \mu \mathrm{s}$

Part I, and this leads to the first ignition site that resides along the centreline of the cavity. Moreover, the temperatures in the Mach stem hot spot (MSHS) are proven high enough that ignition occurs in this hot spot as well, at a time less than half (both for 2D and for 3D simulations) the time required for the ignition of the neat material. By studying the maximum percentage burnt in the two centreline hot spots (CHS) and Mach stem hot spot (MSHS), we observed that the burning in the CHS is, at these timescales, always higher than in the MSHS. However, the maximum burning of the CHS grows as $\sqrt{x}$ while the maximum burning in the MSHS grows linearly. As a result, the growth rate of the maximum burning in the CHS is higher than in the MSHS at first, but this is reversed at late times.

By comparing two- and three-dimensional simulations, we identify the change in topology of the hot spots due to the third dimension. The faster jet (1.4 times faster) in the 3D case results in an earlier collapse of the cavity $(\approx 0.5 \mu \mathrm{s})$ and subsequent hot spot generation compared to $2 \mathrm{D}$. The ignition in the BHS in the 3D case occurs between 3.5 and $4 \mu \mathrm{s}$ and in the MSHS between 5.5 and $6 \mu \mathrm{s}$. Effectively the ignition is observed earlier in the $3 \mathrm{D}$ case by $0.5 \mu \mathrm{s}$, which is the amount of time by which the jet impact occurs earlier in 3D compared to $2 \mathrm{D}$. In the $3 \mathrm{D}$ scenario, the temperatures can reach values of more than three times higher than the postshock temperatures and in the 2D scenario more than twice the post-shock temperature. This leads to a higher percentage of maximum immediate burn of the material upon collapse in $3 \mathrm{D}(59 \%)$ compared to $2 \mathrm{D}(11 \%)$. The growth of the burning follows a similar trend, however, in the two cases.

By comparing inert and reacting simulations, we conclude that the effect of the reaction on the topology of the hot spots is negligible, whereas a large, additive effect on the temperature field is observed. We examine the maximum temperature in the centreline hot spots (CHS) and the MSHS in both inert and reactive scenarios. We demonstrate that after the birth of the hot spot, the maximum temperature in the reactive case is increasing, as expected since the shock waves support the reactions and vice versa. In contrast, the maximum temperature in the inert case is decreasing and the shock waves are not supported. An interesting feature observed is the super- position of waves emanating from the two lobes along the cavity centreline, leading to an additional short-lived maximum temperature peak in both cases.

The maximum temperatures describing the relative "strength" of the CHS and MSHS are studied as well. In the timescales considered, both in the inert and reactive scenarios, the CHS always exhibits higher temperatures than the MSHS. It is interesting to note that the temperatures of the MSHS in the reactive scenario are comparable to the temperatures in the CHS in the inert simulations.

The maximum temperature growth of the hot spots is also studied. Comparing the inert and reactive simulations, we observe that the rate of increase in maximum nitromethane temperature is positive for the reactive configuration but not in the inert case, for both the CHS and the MSHS. Comparing the rate of increase in maximum nitromethane temperature between the two hot spots, it is observed that the rate of increase in the MSHS in the inert case is always higher than in the CHS (except at the birth moment of the CHS, i.e., upon the collapse of the cavity). In the reactive case, however, this is not true. This is likely to have implications in configurations with multiple cavities collapsing in reactive media.

Acknowledgements This project was kindly funded by Orica Limited. The authors also benefited from conversations with A. Minchinton, S.K. Chan and I.J. Kirby.

Open Access This article is distributed under the terms of the Creative Commons Attribution 4.0 International License (http://creativecomm ons.org/licenses/by/4.0/), which permits unrestricted use, distribution, and reproduction in any medium, provided you give appropriate credit to the original author(s) and the source, provide a link to the Creative Commons license, and indicate if changes were made.

\section{References}

1. Michael, L., Nikiforakis, N.: Generation of temperature hot spots in liquid nitromethane. Part I: inert case. Shock Waves (2018). doi:10. 1007/s00193-018-0802-8

2. Bourne, N., Field, J.: Bubble collapse and the initiation of explosion. Proc. R. Soc. Lond. Ser. A Math. Phys. Sci. 435(1894), 423-435 (1991). doi:10.1098/rspa.1991.0153 
3. Bourne, N., Field, J.: Shock-induced collapse and luminescence by cavities. Philos. Trans. R. Soc. Lond. Ser. A Math. Phys. Eng. Sci. 357(1751), 295-311 (1999). doi:10.1098/rsta.1999.0328

4. Bourne, N., Milne, A.: On cavity collapse and subsequent ignition. In: Proceedings of the Twelfth Symposium (International) on Detonation, pp. 213-219. Office of Naval Research (2002)

5. Bourne, N., Milne, A.: The temperature of a shock-collapsed cavity. Proc. R. Soc. Lond. Ser. A Math. Phys. Eng. Sci. 459(2036), 18511861 (2003). doi:10.1098/rspa.2002.1101

6. Michael, L., Nikiforakis, N.: The temperature field around collapsing cavities in condensed phase explosives. In: 15th International Detonation Symposium, pp. 60-70. Office of Naval Research (2014)

7. Kapila, A., Schwendeman, D., Gambino, J., Henshaw, W.: A numerical study of the dynamics of detonation initiated by cavity collapse. Shock Waves 25(6), 545-572 (2015). doi:10.1007/ s00193-015-0597-9

8. Tran, L., Udaykumar, H.: Simulation of void collapse in an energetic material, Part 2: Reactive case. J. Propuls. Power 22(5), 959-974 (2006). doi:10.2514/1.13147

9. Rai, N.K., Schmidt, M.J., Udaykumar, H.: Collapse of elongated voids in porous energetic materials: Effects of void orientation and aspect ratio on initiation. Phys. Rev. Fluids 2(4), 043201 (2017). doi:10.1103/PhysRevFluids.2.043201

10. Rai, N.K., Schmidt, M.J., Udaykumar, H.: High-resolution simulations of cylindrical void collapse in energetic materials: Effect of primary and secondary collapse on initiation thresholds. Phys. Rev. Fluids 2(4), 043202 (2017). doi:10.1103/PhysRevFluids.2.043202

11. Michael, L., Nikiforakis, N.: A hybrid formulation for the numerical simulation of condensed phase explosives. J. Comput. Phys. 316, 193-217 (2016). doi:10.1016/j.jcp.2016.04.017

12. Nikiforakis, N., Clarke, J.: Numerical studies of the evolution of detonations. Math. Comput. Model. 24(8), 149-164 (1996). doi:10. 1016/0895-7177(96)00147-1

13. Saurel, R., Petitpas, F., Berry, R.: Simple and efficient relaxation methods for interfaces separating compressible fluids, cavitating flows and shocks in multiphase mixtures. J. Comput. Phys. 228(5), 1678-1712 (2009). doi:10.1016/j.jcp.2008.11.002

14. Hardesty, D.: An investigation of the shock initiation of liquid nitromethane. Combust. Flame 27, 229-251 (1976). doi:10.1016/ 0010-2180(76)90026-2

15. Tarver, C., Urtiew, P.: Theory and modeling of liquid explosive detonation. J. Energ. Mater. 28(4), 299-317 (2010). doi:10.1080/ 07370651003789317

16. Ripley, R., Zhang, F., Lien, F.: Detonation interaction with metal particles in explosives. In: 13th International Detonation Symposium (2006)

17. Stephen, A., Sheffield, A., Datterbaum, D., Engelke, R., Alcon, R., Crouzet, B., Robbins, D., Stahl, D., Gustavsen, R.: Homogeneous shock initiation process in neat and chemically sensitized nitromethane. In: 13th International Detonation Symposium, pp. 401-409. Office of Naval Research (2006)
18. Peugot, F., Sharp, M.W., The Participants of the NIMIC Shock Modeling Group: NIMIC nations collaborative efforts in shock modeling, reactive models for hydrocodes: past, present and future. Technical Report, National Atlantic Treaty Organization, Brussels, Belgium (2002)

19. Shaw, R., Decarli, P., Ross, D., Lee, E., Stromberg, H.: Thermal explosion times of nitromethane, perdeuteronitromethane, and six dinitroalkanes as a function of temperature at static high pressures of 1-50 kbar. Combust. Flame 35, 237-247 (1979). doi:10.1016/ 0010-2180(79)90029-4

20. Kipp, M., Nunziato, J.: Numerical simulation of detonation failure in nitromethane. Technical Report, Sandia National Laboratories, Albuquerque, NM, USA (1981)

21. Menikoff, R., Shaw, M.S.: Modeling detonation waves in nitromethane. Combust. Flame 158(12), 2549-2558 (2011). doi:10.1016/j.combustflame.2011.05.009

22. Arienti, M., Morano, E., Shepherd, J.: Shock and detonation modeling with the Mie-Grüneisen equation of state. Technical Report, Graduate Aeronautical Laboratories, California Institute of Technology (2004)

23. Dattelbaum, D., Sheffield, S., Stahl, D., Dattelbaum, A., Elert, M., Furnish, M., Anderson, W., Proud, W., Butler, W.: Influence of hot spot features on the shock initiation of heterogeneous nitromethane. AIP Conf. Proc. 1195, 263 (2010). doi:10.1063/1.3295119

24. Bates, K., Nikiforakis, N., Holder, D.: Richtmyer-Meshkov instability induced by the interaction of a shock wave with a rectangular block of $\mathrm{SF}_{6}$. Phys. Fluids 19, 036101 (2007). doi:10.1063/1. 2565486

25. Sheffield, S., Engelke, R., Alcon, R., Gustavsen, R., Robins, D., Stahl, D., Stacy, H., Whitehead, M.: Particle velocity measurements of the reaction zone in nitromethane. In: 12th International Detonation Symposium, pp. 159-166. Office of Naval Research (2002)

26. Berke, J., Shaw, R., Tegg, D., Seely, L.: Shock initiation of nitromethane, methyl nitrite and some bis difluoramino alkanes. In: 5th Symposium (International) on Detonation, p. 237-246. Office of Naval Research (1970)

27. Chaiken, R.: Correlation of shock pressure, shock temperature, and detonation induction time in nitromethane. In: Proceedings of the High Dynamic Pressure Symposium, Commissariat d'Energie Atomique, Paris, France (1978)

28. LeVeque, R.: Nonlinear conservation laws and finite volume methods for astrophysical fluid flow. In: Leveque, R.J., Mihalas, D., Dorfi, E.A., Mueller, E. (eds.) Computational Methods for Astrophysical Fluid Flow, 27th Saas-Fee Advanced Course Lecture Notes, Edited by O. Steiner and A. Gautschy. Springer, Berlin (1998)

29. Lee, J., Higgins, A.: Comments on criteria for direct initiation of detonation. Philos. Trans. R. Soc. Lond. A Math. Phys. Eng. Sci. 357(1764), 3503-3521 (1999). doi:10.1098/rsta.1999.0506 